TRANSACTIONS OF THE

AMERICAN MATHEMATICAL SOCIETY

Volume 358, Number 5, Pages 2291-2334

S 0002-9947(05)03839-0

Article electronically published on December 20, 2005

\title{
CANARD SOLUTIONS AT NON-GENERIC TURNING POINTS
}

\author{
PETER DE MAESSCHALCK AND FREDDY DUMORTIER
}

\begin{abstract}
This paper deals with singular perturbation problems for vector fields on 2-dimensional manifolds. "Canard solutions" are solutions that, starting near an attracting normally hyperbolic branch of the singular curve, cross a "turning point" and follow for a while a normally repelling branch of the singular curve. Following the geometric ideas developed by Dumortier and Roussarie in 1996 for the study of canard solutions near a generic turning point, we study canard solutions near non-generic turning points. Characterization of manifolds of canard solutions is given in terms of boundary conditions, their regularity properties are studied and the relation is described with the more traditional asymptotic approach. It reveals that interesting information on canard solutions can be obtained even in cases where an asymptotic approach fails to work. Since the manifolds of canard solutions occur as intersection of center manifolds defined along respectively the attracting and the repelling branch of the singular curve, we also study their contact and its relation to the "control curve".
\end{abstract}

\section{INTRODUCTION}

Consider a singularly perturbed vector field on a 2-dimensional manifold, depending on a small parameter $\varepsilon$. A typical question is to what extent solutions of the perturbed vector field remain "comparable" to solutions of the unperturbed vector field $(\varepsilon=0)$. Geometrically speaking, the question can be formulated in terms of the persistence of invariant manifolds under perturbation. It becomes interesting if the unperturbed vector field has a curve of singularities (a "critical curve") that disappears after perturbation. The dynamics of the phase portrait can then be decomposed in a slow and a fast part. The fast dynamics are governed by the attraction towards (or repulsion away from) the critical curve in the unperturbed vector field. The slow dynamics appear because the curve of singularities perturbs, so near this curve a slow drift along it is observed. Both laws play a role for orbits of points that lie very close to the critical curve, and both will compete for dominance. The geometric theory of Fenichel states that orbits of perturbed vector fields follow pieces of slow dynamics for some time and then revert to pieces of the fast dynamics for some time, or vice versa, depending on whether the normal hyperbolicity is repelling or attracting at the moment of reversion. Fenichel's theorem is valid under certain generic conditions (of which the most notable one is the normal hyperbolicity along the curve of singularities). Apparently, in points where

Received by the editors May 19, 2003 and, in revised form, August 30, 2004.

2000 Mathematics Subject Classification. Primary 34E15, 34E20, 34C26, 34A12; Secondary 34D15, 37G15, 34B99.

Key words and phrases. Singular perturbations, canard solutions, degenerate turning point, center manifolds, normal forms, blow up of families.

(C)2005 American Mathematical Society Reverts to public domain 28 years from publication 
normal hyperbolicity is lost, the dynamics can be more complicated. This is because attraction can change into repulsion on crossing such "contact points". The competition between slow and fast dynamics is thus most apparent in points like these. One might expect that in situations where orbits follow the slow movement, the orbits will revert to orbits following fast dynamics immediately after crossing a contact point, because of the repulsive behaviour after this point. On the contrary, sometimes the orbits will keep on following the critical curve for some time, before the exchange of dominance occurs. Contact points with that property could be called "turning points" to make a distinction with the more common "jump point" which one encounters in relaxation oscillations.

When the family of vector fields has other parameters, we can try - under rather general conditions - to give a regular condition on $\varepsilon$ and on the other parameters so that the exchange of stability occurs at exactly the manifold in parameter space given by the condition. This process will create what is commonly known as "overstable solutions", or "canard solutions". In the literature, a clear distinction is made between these two names: the term overstable solution is used in the complex setting, whereas real solutions are referred to as canard solutions. In the literature, several successful methods have been brought forward to handle the existence of both overstable solutions and canard solutions. We mention the technique of matching inner and outer solutions (Eckhaus, Mishchenko, Kolesov, Rozov, ....), non-standard analysis (Diener et al.) and also complex analytic techniques (Sibuya, Schäfke, Ramis, Canalis-Durand and many others). Lately, serious progress has been made in applying analytic techniques to singularly perturbed problems, and a number of the results that are proved in this work have already been proved using these techniques. But the analytic study does not completely cover the dynamics on the real axis. We present some examples where real canard solutions are created, that cannot be complexified to canard solutions in full complex neighbourhoods (or so-called overstable solutions).

The method we use here is based on the construction of center manifolds and the use of $C^{k}$ normal forms, as in DR and DR2. It has also been used by Krupa and Szmolyan in e.g. KS1, KS2], KS3] and [KS4]. The key element is the family blow up - a technique of rescaling variables in a geometrical way. Because of this, the constructed solutions will be smooth in the blow up space. We present conditions under which a blow down of the center manifolds is possible. We extract some consequences from it and relate these to treatments of a different nature like the traditional matching between "inner" (inside the blow up locus) and outer solutions (in original coordinates) or the resummation.

Our major contribution lies in the generality of our results. Where up to now the geometric analysis of turning points (like in $[\overline{D R}]$ ) was restricted to the generic case, we consider here a generalization to non-generic turning points. A specific class of more degenerate systems is precisely described in Theorem 5. In Theorem 4 we show that our results can be applied to what we could call the generic turning point. These examples are defined in the plane, but our results apply, as the description shows, to systems on 2-manifolds. All results are valid for vector fields of class $C^{r}, r$ sufficiently large.

A second part of this text deals with the angle between center manifolds, defined on different sides of the contact point. In fact, the entire canard phenomenom can be explained geometrically by looking at how those two different families of center 
manifolds intersect. Orbits following the critical curve in positive time, gathered in these invariant (center) manifolds, cross the contact point and intersect with orbits following the critical curve in negative time. Any connection between these manifolds results in canard solutions, so the intersection is crucial in our study. If the manifolds intersect transversally, a straightforward adaption of the techniques in $[\mathrm{DR}$, results in a formula for the angle. We generalize these computations by providing recursive formulas for calculating the first nonzero higher order angle, besides describing its relation with the graph of the control curve.

\section{Fundamental notions And Statement of Results}

In this section, we will put some constraints on the vector fields under study. We have tried to write these constraints as much as possible in a coordinate free manner. Later, we give important classes of vector fields where these conditions are met. But before stating precise conditions and results, we quickly review some relevant notions.

Definition 1. A critical curve of a singularly perturbed family of vector fields $X_{\varepsilon}$ on a 2-manifold $M$ is a curve of singularities of the reduced vector field $X_{0}$. In this paper, we will regard the critical curve as a curve in the manifold $M \times\{0\}=\{\varepsilon=0\}$ in a 3-dimensional manifold with boundary $M \times\left[0, \varepsilon_{0}\left[\right.\right.$ for some $\varepsilon_{0}>0$. If the vector field depends on other parameters $\lambda$ we will still call it - with abuse of language the critical curve.

In our study we will also use a special parameter - denoted by $a$ - that will essentially be a parameter breaking the critical manifold in a regular way. In a moment we will give a precise definition of it. If $M$ is the plane $\mathbb{R}^{2}$, this would mean we consider smooth families of vector fields

$$
X_{\varepsilon, a, \lambda}:\left\{\begin{aligned}
\dot{x} & =f(x, y, \varepsilon, a, \lambda) \\
\dot{y} & =\varepsilon g(x, y, \varepsilon, a, \lambda)
\end{aligned}\right.
$$

with singular parameter $\varepsilon \in \mathbb{R}^{+}$, and where $(a, \lambda) \in \mathbb{R} \times \Lambda\left(\Lambda \subset \mathbb{R}^{p}\right)$. We could also work with analytic $X_{\varepsilon, a, \lambda}$, and throughout the paper we will try to stress the analyticity or the lack of analyticity of the results in case of analytic $X_{\varepsilon, a, \lambda}$. We assume that $X_{0,0, \lambda}$ has a curve of singularities $\gamma$. This curve may depend on $\lambda$, but we will not keep this dependence in the notation of $\gamma$. The dependence on $\lambda$ is not entirely unconditional; obvious bifurcations in the shape of the curve should be avoided. We in fact ask $\gamma=\gamma_{\lambda}$ to be a trivial $\lambda$-family of simple curves; for a precise statement we refer to a remark after Assumption 3.

The paper will deal with simple critical curves with a single contact point. By "simple" we mean that the curve can be obtained as an image of a $C^{\infty}$ embedding of $[0,1]$. It is "slow" since it consists of singularities of the vector field under consideration; $\gamma$ contains a point $p_{\gamma}$ not lying at its endpoint, with the property that $p_{\gamma}$ divides $\gamma$ into two parts $\gamma_{-}$and $\gamma_{+}$with both $\gamma_{-} \cup\left\{p_{\gamma}\right\}$ and $\gamma_{+} \cup\left\{p_{\gamma}\right\}$ simple critical curves. We orient $\gamma$ in a way that $\gamma_{+}$comes after $\gamma_{-}$. The fact that $p_{\gamma}$ is a simple contact point means that $X_{0}$ is normally attracting at all points of $\gamma_{-}$and normally repelling at all points of $\gamma_{+}$.

Assumption 1 (Admissible chart): There exists a (possibly $\lambda$-dependent) chart of $M$ in the neighbourhood of $p_{\gamma}$ so that in this chart the contact point is the origin $(x, y)=(0,0)$. Writing the vector field in this chart as in (1), the critical curve is given by $\gamma=\{(x, y) \mid f(x, y, 0,0, \lambda)=0\}$. The origin 
divides $\gamma$ in two pieces $\gamma_{-}$and $\gamma_{+}$. Along $\gamma \backslash\{(0,0)\}$ we suppose that both $\frac{\partial f}{\partial x}$ and $\frac{\partial f}{\partial y}$ are nonzero, and that $\gamma_{-}$is a graph over the negative real $x$-axis, and $\gamma_{+}$is a graph over the positive real $x$-axis.

A chart where these conditions are met is called an admissible chart.

Assumption 2 deals with the possibility of passage near the critical curves. Let us focus on $\gamma_{-}$; with respect to the repelling part $\gamma_{+}$we require analogous properties.

The unperturbed vector field $X_{0}$ on $M$ is normally attracting at all points of $\gamma_{-}$. Let $p$ be a point of $\gamma_{-}$, and consider the vector field $X=X_{0}+0 \frac{\partial}{\partial \varepsilon}$ on $M \times\left[0, \varepsilon_{0}[\right.$. Because of the center manifold theorem we can find locally around $p$ (possibly parameter-dependent) 2-dimensional invariant center manifolds $W_{p}$ that are at least $C^{1}$. Let us consider such a center manifold. Although actually being a manifold with boundary in $M \times\left[0, \varepsilon_{0}\right.$ [, let us - by abuse of language - call it a manifold. Essentially, we want the dynamics on the center manifolds to be topologically equivalent to the dynamics of a model differential equation

$$
\varepsilon \frac{\partial}{\partial x}+0 \frac{\partial}{\partial \varepsilon} \text {. }
$$

More precisely, we want the existence of a $C^{1}$ embedding

$$
\varphi:[0,1]^{2} \rightarrow W_{p}:(x, \varepsilon) \mapsto \varphi(x, \varepsilon),
$$

so that

(i) $\varphi\left([0,1]^{2}\right)$ is a neighbourhood of $p$ inside $W_{p}$;

(ii) $\varphi([0,1] \times\{0\}) \subset\{\varepsilon=0\}$;

(iii) $\left.\varphi\right|_{\varepsilon=0}$ is orientation-preserving for the standard orientation on the $x$-axis and the chosen orientation on $\gamma$.

(iv) $\varphi$ is a topological equivalence between $\left.X\right|_{W_{p}}$ and the model vector field (2).

To ensure this model behaviour, it suffices to assume

Assumption 2: For any point $p$ of $\gamma_{-} \cup \gamma_{+}$, there exists a sufficiently small neighbourhood $V$ of $p$ in $M \times\left[0, \varepsilon_{0}\right.$ [ so that in $V$ there are no singularities for $\varepsilon>0$. Furthermore, inside center manifolds in $V$, the orientation of the orbits for $\varepsilon>0$ must be compatible to the orientation on $\gamma$.

The compatibility of the orientation of orbits inside center manifolds with the orientation of $\gamma$ means the following: if we take two sections $\sigma_{1}$ and $\sigma_{2}$, transversally cutting $\gamma$ in points with parameter values $r_{1}$ and $r_{2}, r_{1}<r_{2}$ (according to the orientation), then the orbits for $\varepsilon>0$ will also be oriented from $\sigma_{1}$ to $\sigma_{2}$. Assumption 2 does not depend on the chosen center manifold inside $V$.

Note 1. Assumption 2 gives the basis for a possible transition from attracting to repelling regime. In other situations, where the transition is from repelling to attracting, repelling to repelling or attracting to attracting regime, canard solutions are more likely to occur, and the study is easier. Canard solutions occurring from transitions like these are often called "faux canards".

Note 2. In some results we will even allow singularities bifurcating out of $\gamma_{-} \cup \gamma_{+}$, for $\varepsilon>0$, but we will limit ourselves to results concerning invariant manifolds consisting of orbits for which, in the vicinity of $\gamma_{-} \cup \gamma_{+}$, the orientation is compatible with the orientation of $\gamma$. 
Assumption 3 will be a regularity condition, and will be described in terms of an "admissible chart" near the contact point. We will assume that after a single (family) blow up, the vector field will be desingularized in a nice way. Before making this precise, let us recall what a family blow up is, and what the regularity condition means.

At first, a blow up map can be thought of as a singular change of coordinates, like the polar-coordinate mapping

$$
(u, \theta) \mapsto(x, y)=(u \cos \theta, u \sin \theta) .
$$

Another way to denote this map is $(u,(\bar{x}, \bar{y})) \mapsto(u \bar{x}, u \bar{y})$, where $\bar{x}^{2}+\bar{y}^{2}=1$. Now, instead of using coordinates on the circle, we can use charts to rectify parts of the circle. In the above example, near $\bar{x}=1$, we might as well use the coordinate change $(u, \tilde{y}) \mapsto(x, y)=(u, u \tilde{y})$, with $\tilde{y}$ in a fixed domain. On this new chart, the point $\tilde{y}= \pm \infty$ corresponds to $(\bar{x}, \bar{y})=(0, \pm 1)$. In order not to exaggerate in notation, one often uses the symbol $\bar{y}$ for $\tilde{y}$, and one says to work in the $\{\bar{x}=1\}$ chart.

The vector field can be pulled back under the blow up map, and if the origin is a singularity of the original vector field, then the locus $u=0$ will be an invariant set of the pull-back vector field, often even a set of singularities. In the latter case, we desingularize the new vector field by dividing out a positive factor $u^{i}$.

Although desingularization by means of blow up, as the one above, is practical, it can be made more useful by adapting the exponents to the problem under study. If instead of the homogeneous blow up we use for example $(x, y)=\left(u^{2} \bar{x}, u \bar{y}\right)$, with $\bar{x}^{2}+\bar{y}^{2}=1$, or even with $\bar{x}^{2}+\bar{y}^{4}=1$ if advantageous, we sometimes get better results. This generalization gives us the possibility of assigning weights to all the variables, and the weights will in practice be chosen in a way to reach the best desingularization.

An extra possibility when blowing up singularly perturbed differential equations is to include $\varepsilon$ in the list of variables, coming to the notion of family blow up.

If we have a 3 -dimensional family of vector fields $X_{\varepsilon, \lambda}+0 \frac{\partial}{\partial \varepsilon}$, and we want to blow up the origin $(x, y, \varepsilon)=(0,0,0)$, then we use:

$$
\left\{\begin{array}{l}
x=u^{p} \bar{x} \\
y=u^{q} \bar{y} \\
\varepsilon=u^{m} \bar{\varepsilon},
\end{array}\right.
$$

with $(\bar{x}, \bar{y}, \bar{\varepsilon}) \in S^{2}$, and $u \in \mathbb{R}^{+}$. The weights $p, q$ and $m$ are chosen differently for different systems. The best choice can be evident and found without difficulty or can be based on the use of Newton polyhedra; see e.g. [D.

On the 2-sphere, we have the relation $\bar{x}^{2}+\bar{y}^{2}+\bar{\varepsilon}^{2}=1$, often implying the need of working in charts.

Suppose we want to look near the critical curve in these new coordinates, hence in the region $\varepsilon \sim 0$. By Assumption 1, in a well-chosen chart, $\gamma_{-}$is a graph over the negative real $x$-axis, and hence $\gamma_{-}$would be visible in the chart

$$
\left\{\begin{array}{l}
x=-u^{p}, \\
y=u^{q} \bar{y} \\
\varepsilon=u^{m} \bar{\varepsilon} .
\end{array}\right.
$$

This chart is valid for $(\bar{y}, \bar{\varepsilon})$ small, and is called a phase-directional rescaling chart, or simply the $\{\bar{x}=-1\}$ chart. Notice that the trivial foliation $d \varepsilon=0$ is 


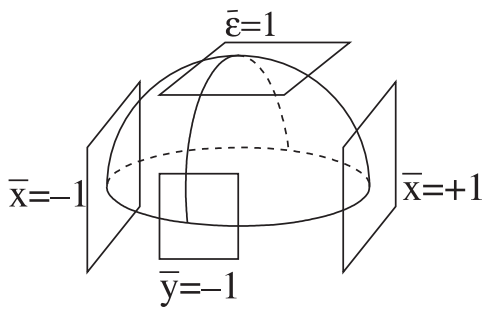

FiguRE 1. Different charts

replaced here by $d\left(u^{m} \bar{\varepsilon}\right)=0$. If the weights are chosen properly, then the preimage of $\gamma_{-}$under the blow up map has a limit for $u \rightarrow 0$. We denote this point with $P_{-}$, and it is in fact the intersection of $\gamma_{-}$with the blow up locus.

Orbits of vector fields in this space will respect the foliation $d\left(u^{m} \bar{\varepsilon}\right)=0$, so as $u$ decreases, $\bar{\varepsilon}$ will increase. Continuing the orbits, we will eventually need to enter a region where $\bar{\varepsilon}$ is no longer small, and where we can bound $|\bar{x}|$ away from 1 . This part is visible in "the chart $\{\bar{\varepsilon}=1\}$ ", commonly known as the family rescaling chart, and the formulas to work in this chart are:

$$
\left\{\begin{array}{l}
x=u^{p} \bar{x}, \\
y=u^{q} \bar{y} \\
\varepsilon=u^{m} .
\end{array}\right.
$$

This chart is valid for $(\bar{x}, \bar{y})$ in a bounded set. In this chart, $u$ is clearly the singular parameter, and we again have a family of vector fields (since $d u=0$ ). This is the traditional chart where "rescaling" is done.

Observe that the $\bar{y}$ coordinate in (5) is not the same as $\bar{y}$ in (4), but intuitively they serve a common purpose, in the sense that they are both a rescaled form of the same $y$ coordinate. It is of course easy to give formulas for the relation between the two expressions of $\bar{y}$.

As $\bar{x}$ gets closer to $+1, \bar{\varepsilon}$ gets closer to 0 , so we will have to leave the $\bar{\varepsilon}=1$ chart. As in the first part, we study this section using the chart:

$$
\left\{\begin{array}{l}
x=+u^{p}, \\
y=u^{q} \bar{y} \\
\varepsilon=u^{m} \bar{\varepsilon} .
\end{array}\right.
$$

Here, the $\gamma_{+}$part of the critical curve will be visible, and as before, if the weights are chosen properly, the preimage of this critical curve will have a well-defined limit point $P_{+}$as $u \rightarrow 0$.

The whole process can be dependent on the extra parameters $\lambda$, which we do not blow up. In the description we also keep $a=0$.

We are now ready for stating precise requirements:

Assumption 3 (Regularity condition): After blowing up at $(0,0,0)$ the $(x, y, \varepsilon)$-variables - in an admissible chart-of $X_{\varepsilon, 0, \lambda}$, we get the following:

The preimages of $\gamma_{-}$and $\gamma_{+}$in the blow up space (including the end points of $\gamma_{ \pm}$on the blow up locus) are normally hyperbolic. Define $P_{ \pm}=$ $\gamma_{ \pm} \cap \Sigma$, where $\Sigma$ is the blow up locus, i.e. the preimage of $(x, y, \varepsilon)=(0,0,0)$ under the blow up map. 
Important remark. The regularity condition is restrictive in the following sense: the blow up weights are chosen independent of $\lambda$, hence in order for the blow up procedure to work for all choices of $\lambda$, the order of degeneracy of the critical curve must not depend on $\lambda$. We have in mind, for example, quadratic contacts where the second order angle changes:

$$
\gamma: y=\lambda x^{2}+O\left(x^{3}\right), \quad \lambda>\lambda_{0}>0,
$$

or contacts of order " $2 n$ ", with a fixed $n$, i.e.,

$$
\gamma: y=\lambda x^{2 n}+O\left(x^{2 n+1}\right), \quad \lambda>\lambda_{0}>0 .
$$

Situations where the order of degeneracy of the critical curve undergoes a bifurcation (for example, $\gamma: y=\lambda x^{2}+x^{4}$, where $\lambda=0$ is inside the parameter space) will generally not satisfy Assumption 3. The way to proceed in these situations is to include $\lambda$ in the family blow up.

The next assumption is the sequel of Assumption 2, but expressed in blow up coordinates which we will introduce. There must be a way to proceed along the "corner". We work in a phase-directional rescaling chart, as in (44). Choosing a section $\left\{u=u_{0}>0\right\}$ means choosing a section in the neighbourhood of the normally hyperbolic part of the critical curve; whereas choosing a section $\left\{\bar{\varepsilon}=\bar{\varepsilon}_{0}>0\right\}$ means choosing a section transversally cutting the blow up locus. As in Assumption 2, we have in mind a model vector field to express the dynamics in center manifolds. First, the existence of center manifolds near $P_{-}$follows from Assumption 3. Choosing a manifold $W$ near $P_{-}$, we essentially want the vector field on $W$ to be equivalent to

$$
-u \bar{\varepsilon} \frac{\partial}{\partial u}+\bar{\varepsilon}^{2} \frac{\partial}{\partial \bar{\varepsilon}} \text {. }
$$

More precisely, let $p=P_{-}$be the end point of $\gamma_{-}$, in a blown up admissible chart, and let $W_{p}$ be a center manifold of $X$ at $p$. We require the existence of a $C^{1}$ embedding

$$
\varphi:[0,1]^{2} \rightarrow W_{p}:(u, \bar{\varepsilon}) \mapsto \varphi(u, \bar{\varepsilon})
$$

so that

(i) $\varphi\left([0,1]^{2}\right)$ is a neighbourhood of $P_{-}$inside $W_{p}$;

(ii) $\varphi([0,1] \times\{0\}) \subset \gamma_{-} \cup\left\{P_{-}\right\}$;

(iii) $\varphi(\{0\} \times[0,1])$ is inside the blow up locus.

(iv) $\left.\varphi\right|_{[0,1] \times\{0\}}$ is orientation-preserving for the standard orientation on the $u$ axis and the negative orientation on $\gamma$.

(v) $\varphi$ is a topological equivalence between $\left.X\right|_{W_{p}}$ and the model vector field (77).

This model behaviour will be ensured by Assumption 2 and the next assumption:

Assumption 4 (Regular corner passage): Let $p=P_{-}$be the end point of $\gamma_{-}$in a blown up admissible chart, then there exists a sufficiently small neighbourhood $V$ of $p$ so that in $V$ there are no singularities for $\bar{\varepsilon}>0$. A similar requirement is made for the end point $P_{+}$of $\gamma_{+}$.

Assumption 5 (Connection condition): Under the conditions of Assumption 3 , there is a heteroclinic connection $\Gamma($ for $a=0)$ on the blow up locus $\Sigma$ connecting $P_{-}$to $P_{+}$. We assume that this connection consists of one orbit going from $P_{-}$to $P_{+}$. 
Of course, it would be interesting to see what can happen if we let a parameter tend to the boundary of the parameter set where Assumption 5 is satisfied. Possibly, a saddle-node may appear on the connection, or, the connection may be a curve of singularities in the limit. At first, we will focus on the case where there is a connection without singularities, but the techniques are general enough to be used in more degenerate cases; see the examples for such a generalization in the normally hyperbolic passage.

Let us now precisely describe the role of $a$, starting by describing the role of a regular breaking parameter in the case that $\gamma$ remains a critical manifold for the vector fields $X_{\varepsilon, a, \lambda}$ with $a \neq 0$. To focus on the specificity of this case let us write $a=A$. In that case the blow up in $(x, y, \varepsilon)$-space cannot only be applied for $A=0$ but also for the fields with $A \neq 0$. Due to Assumption 3 we recover, for $A=0$, in the blow up the points $P_{ \pm}$, that will persist as normally hyperbolic points for small values $A \neq 0$. Also the invariant manifolds of respectively $P_{-}$and $P_{+}$inside the blow up locus $\{\varepsilon=0\}$ will persist. We know from Assumption 5 that they form a heteroclinic connection for $A=0$. In the family rescaling chart and inside $\{\varepsilon=0\}$ we can choose a section $\sigma$ transverse to the flow of the blow up vector field. We choose a regular parameter $z$ on $\sigma$ and we denote by $z_{ \pm}(A, \lambda)$ the intersection with $\sigma$ of the invariant manifolds of respectively $P_{ \pm}$. By Assumption 5 , we know that $z_{-}(0, \lambda)=z_{+}(0, \lambda)$.

Definition 2. We say that $A$ is a regular breaking parameter if

$$
\rho(\lambda):=\frac{\partial}{\partial A}\left(z_{-}-z_{+}\right)(0, \lambda) \neq 0 .
$$

This definition does not depend on the choice of the regular coordinate $z$, nor on the transverse section $\sigma$.

Assumption 6 (Breaking parameter): Using the family rescaling chart expressed in (5), there exists some $n \in \mathbb{N}$ such that $A:=a / u^{n}$ (hence $\left.a=A \varepsilon^{n / m}\right)$ is a regular breaking parameter.

The idea is that the family $X_{\varepsilon, a, \lambda}$ is replaced by a subfamily $X_{\varepsilon, A \varepsilon^{n / m}, \lambda}$, but in this subfamily we know that the critical curve $\gamma$ of $X_{\varepsilon, 0, \lambda}$ persists to a critical curve of $X_{\varepsilon, A \varepsilon^{n / m}, \lambda}$ with $A \neq 0$.

The presentation in this paper is adapted to the study of the so-called canard solutions. It means that we stay in a region in parameter space outside, where no such solutions can exist. This is reflected in the rescaling

$$
(a, \varepsilon)=\left(u^{n} A, u^{m}\right),
$$

as used to express Assumption 6. In fact, if one wants to use family blow up to make a study in a complete neighbourhood of $(0,0)$ in the $(a, \varepsilon)$-plane, the way to proceed is first to make a blow up in the parameter plane by writing

$$
(a, \varepsilon)=\left(v^{k} A, v^{\ell} E\right),
$$

for $A^{2}+E^{2}=1$ (or equivalently working with charts $E=+1$ or $A= \pm 1$ ). Thereafter one continues with blow up in the $(x, y, v)$-space:

$$
(x, y, v)=\left(u^{p} \bar{x}, u^{q} \bar{y}, u \bar{\varepsilon}\right),
$$

with $\bar{x}^{2}+\bar{y}^{2}+\bar{\varepsilon}^{2}=1$ (or equivalently working with charts). It of course leads to the same result under the condition $E=1$. 
In Sections 3.2, 4 and 5 we will hence write system (10) as

$$
X_{v, A, \lambda}:\left\{\begin{aligned}
\dot{x} & =f(x, y, v, A, \lambda), \\
\dot{y} & =v g(x, y, v, A, \lambda)
\end{aligned}\right.
$$

to emphasize that we might already have written the original $(a, \varepsilon)$ as $(a, \varepsilon)=$ $\left(v^{k} A, v^{\ell}\right)$ for some $(k, \ell) \in \mathbb{N}^{2}$. We can hence suppose that $X_{0, A, \lambda}$ has a curve of singularities through the origin for all $(A, \lambda)$ under consideration.

As an example, consider the Van der Pol system

$$
\left\{\begin{array}{l}
\dot{x}=y-\frac{x^{2}}{2}-\frac{x^{3}}{3} \\
\dot{y}=\varepsilon(a-x)
\end{array}\right.
$$

In this example, $a$ is not a regular breaking parameter; however, in [DR] it is shown that $a / \sqrt{\varepsilon}$ is a regular breaking parameter. Hence, in (11) one can write

$$
a=v A, \quad \varepsilon=v^{2} E,
$$

with $A^{2}+E^{2}=1$. Being interested in $A \sim 0$, we can consider the chart $E=1$, and check the assumptions for the parameters $(v, A)$ instead of $(\varepsilon, a)$.

Important remark. Assumption 2, the second part of Assumption 5 and Assumption 4 are "open" assumptions, i.e., we could restrict the parameter set $(A, \lambda)$ to an open subset where these conditions are satisfied. It would be interesting to know what happens if $(A, \lambda)$ tends to the boundary of this set, i.e., a singularity could appear on the slow dynamics, or on the heteroclinic connection, or even more degenerate phenomena could occur. In extremis, the connection on the blow up locus could consist of singular points, with possible loss of normal hyperbolicity at some point!

To formulate the results, we need some definitions.

Definition 3. A "simple passage" turning point is a contact point satisfying the properties described in Assumptions 1-6 above.

Definition 4. The basin of attraction of $\gamma_{-}$is the set of points in the manifold $M$ for which the orbit in positive time under the unperturbed vector field $X_{0,0, \lambda}$ has its $\omega$-set in $\gamma_{-}$. A similar definition holds for the basin of repulsion of $\gamma_{+}$, using the $\alpha$-set.

Definition 5. Let $\Sigma$ be a smooth curve in $M \times\left[0, \varepsilon_{0}\right.$ [, possibly depending on some extra parameters. Assume that this curve is a graph in $\varepsilon \geq 0$. The saturation of $\Sigma$ is defined as the topological closure of the union of all orbits (w.r.t. the extended vector field $X_{\varepsilon, a, \lambda}+0 \frac{\partial}{\partial \varepsilon}$ ) of points of $\Sigma$. The need to take a topological closure becomes clear if one considers the limit point of $\Sigma$ as $\varepsilon \rightarrow 0$ : the orbits become singular for this limit point.

The main result is Theorem 2, but a first preliminary result is the existence of canard solutions, with arbitrary boundary conditions. Two boundary conditions are chosen as follows: take a smooth curve $\Sigma_{-}$, transverse to the manifold $\{\varepsilon=0\}$ and so that the end point $b_{-}$in $\{\varepsilon=0\}$ is inside the basin of attraction of $\gamma_{-}$, and take a smooth curve $\Sigma_{+}$transverse to $\{\varepsilon=0\}$ so that the end point $b_{+}$is inside the basin of repulsion of $\gamma_{+}$. Theorem 1 states that we can write the parameter $a$ in terms of $\varepsilon$ in a way that the saturation in forward time of $\Sigma_{-}$coincides with the saturation in backward time of $\Sigma_{+}$, thereby creating a manifold of canard solutions for (11). The canard solutions are global in nature in this approach. 
The saturation of $\Sigma_{-}$contains the limit point in $\gamma_{-}$of the orbit of $b_{-}$. We will call this limit point a corner point of the saturation of $\Sigma_{-}$and denote it by $c_{-}$. Similarly, a corner point $c_{+}$for the saturation of a curve $\Sigma_{+}$can be introduced. We need one more definition.

Definition 6. An admissible entry boundary curve $\Sigma_{-}$is a curve in $M \times\left[0, \varepsilon_{0}[-\right.$ space (possibly $(a, \lambda)$-dependent), that is, a graph $\varepsilon \mapsto s_{-}(\varepsilon) \in M$. We assume that $s_{-}$is $C^{\infty}$ for $\varepsilon>0$, and $C^{0}$ at $\varepsilon=0$. Furthermore, we assume

$$
\forall n \in \mathbb{N}, \exists N \in \mathbb{N}: \frac{\partial^{n}}{\partial \varepsilon^{n}} s_{-}(\varepsilon)=O\left(\varepsilon^{-N}\right), \quad \text { as } \varepsilon \rightarrow 0 .
$$

Similarly, an admissible exit boundary curve can be defined.

This definition allows graphs that are $C^{\infty}$ at $\varepsilon=0$, but also graphs like $\varepsilon \mapsto$ $\varepsilon \log \varepsilon$, graphs that are $C^{\infty}$ in $\varepsilon^{1 / r}$ for some $r>0$, etc.

Theorem 1. Let $X_{\varepsilon, a, \lambda}$ be a vector field on a 2-manifold with a simple passage turning point. Let $\Sigma_{ \pm}$be admissible entry/exit boundary curves. Then for some $m \in \mathbb{N}$ and for $\varepsilon \in\left[0, \varepsilon_{0}\left[\right.\right.$ with $\varepsilon_{0}>0$ sufficiently small, there exists a unique smooth curve $a=\mathcal{A}\left(\varepsilon^{1 / m}, \lambda\right)$ so that $\mathcal{A}(0, \lambda)=0$ and so that the saturation of $\Sigma_{-}$along $X_{\varepsilon, \mathcal{A}\left(\varepsilon^{1 / m}, \lambda\right), \lambda}$ forms a manifold with boundary of canard solutions containing $\Sigma_{+}$ as well. The manifold with boundary is smooth in the blow up space, everywhere excep1 at the two corner points $c_{ \pm}$defined above. The $\infty$-jet of $\mathcal{A}(u, \lambda)$ w.r.t. $u$ is independent of the chosen admissible entry/exit boundary curves.

Remark 1. A curve $a=\mathcal{A}\left(\varepsilon^{1 / m}, \lambda\right)$, as in the statement of Theorem 10 is called a control curve, or a canard line. It depends on the choice of $\Sigma_{ \pm}$. However, two different control curves have an infinite contact at $\varepsilon=0$, uniformly in $\lambda$.

Remark 2. The smoothness of the control curve and of the manifolds will be in terms of the rescaled variables, due to the rescaling. This effect is most visible in the control curve; this curve will in general only be smooth in $\varepsilon^{1 / m}$ for some $m \in \mathbb{N}$ depending on the blow up construction.

Remark 3. This theorem has strong implications on the orbits of points on $\Sigma_{-}$, for $\varepsilon \sim 0$. The corner points are essentially the points where the change of dominance takes place. Following the fast dynamics, a point of $\Sigma_{-}$fastly moves towards a small neighbourhood of the critical curve $\gamma_{-}$, near the corner point $c_{-}$, then staying close to $\gamma_{-}$slowly moves over the contact point towards the repelling part of the critical curve, and near $c_{+}$again moves quickly away from $\gamma_{+}$finally reaching $\Sigma_{+}$.

Remark 4. The manifolds with boundary in Theorem 1, and other manifolds with boundary will be referred to as manifolds.

Remark 5. If two different sets of boundary curves $\Sigma_{ \pm}$and $\Sigma_{ \pm}^{\prime}$ are taken, then the two manifolds are infinitely tangent to each other between the corner point $c_{-}$or $c_{-}^{\prime}$ (whichever is closer to the contact point) and the corner point $c_{+}$or $c_{+}^{\prime}$. Also, the control curves are infinitely tangent to each other.

A second result concerns the possibility of blowing down the invariant manifold and getting smoothness in the original phase space. When blowing down, it is a priori possible to loose differentiability. (Written in polar coordinates, a cone for

\footnotetext{
${ }^{1}$ If the boundary curves are nonsmooth at their base points, then the smoothness is of course also lost along the orbits of these base points.
} 
example is differentiable w.r.t. $(r, \theta)$, but it is not a differentiable object in cartesian coordinates.) In Section 8.1 we will show an example of such a phenomenon for fields $X_{\varepsilon, a, \lambda}$ satisfying all assumptions which we made. So let us ask ourselves the question when the invariant manifolds blow down to differentiable objects. Obviously, a necessary condition is the existence of a Taylor expansion in the origin. The following theorem states that this is in fact also a sufficient condition.

Theorem 2. Let $X_{\varepsilon, a, \lambda}$ be a vector field on a 2-manifold with a simple passage turning point. Because of this, we already know that the blow down of the invariant manifolds from Theorem 1 are in an admissible chart near the contact point graphs $y=\Psi(x, \varepsilon, \lambda)$. Assume that there exist formal power series

$$
\hat{a}=\sum_{n=0}^{\infty} a_{n}(\lambda) \varepsilon^{n}, \quad \hat{y}=\sum_{n=0}^{\infty} y_{n}(x, \lambda) \varepsilon^{n}
$$

so that $y_{n}$ is smooth in a uniform neighbourhood of $x=0$, and so that $\hat{y}$ is formally invarian 2 under $\hat{X}_{\varepsilon, \hat{a}, \lambda}$, then $\Psi$ will be smooth in a neighbourhood of the origin. The infinite jet $j_{\infty}(\Psi)$ will coincide with $\hat{y}$, and also $j_{\infty}(\mathcal{A})$ will coincide with $\hat{a}$.

By this theorem, it is also clear that the canard solutions constructed by means of analytic techniques coincide with the invariant manifolds from Theorem 1 . It is well known that such formal solutions are unique, if they exist, under the assumptions that we made.

The invariant manifolds in Theorem 1 are constructed by connecting "center manifolds" along $\gamma_{-}$to center manifolds along $\gamma_{+}$. This concept is important in order to understand the next theorem, so let us recall a few notions.

Definition 7. A "center manifold" for the system (1) is the saturation of a local center manifold at a normally hyperbolic point on the critical curve. The saturation may define an invariant manifold up to the contact point, or more specifically, up to a part of the blow up locus. Thus, two classes of center manifolds exist: center manifolds along $\gamma_{-}$and along $\gamma_{+}$. The quotes around center manifolds make it clear that this is not a center manifold at the contact point. It is important to notice that the center manifolds depend regularly on $A$, unlike the manifolds of canard solutions, where $A$ has already been expressed in terms of $\varepsilon$.

The idea is that the left center manifolds and the right center manifolds can be compared when intersecting both with a section transversally cutting the blow up locus. More precisely, in the family rescaling chart we can e.g. take a section $T:\{\bar{x}=0\}$ and look at the intersection of the left and right center manifolds with $T$. Choosing coordinates $z$ on $\sigma:=T \cap\{u=0\}$, we can use $(z, u)$ as coordinates on $T$. The intersection of the left and right center manifolds with $T$ are hence graphs

$$
z=\zeta_{ \pm}(u, A, \lambda)
$$

Notice that

$$
\zeta_{ \pm}(0, A, \lambda)=z_{ \pm}(A, \lambda)
$$

where $z_{ \pm}$is the graph in (8).

Intuitively, the next theorem states that the angle between $\zeta_{-}$and $\zeta_{+}$is proportional to the angle of the control curve of the canard solutions.

\footnotetext{
${ }^{2}$ We say that $y=\psi(x, \varepsilon)$ is formally invariant under $X_{\varepsilon}:\{\dot{x}=f(x, y, \varepsilon), \dot{y}=g(x, y, \varepsilon)\}$ if the infinite jet of $f(x, \psi(x, \varepsilon), \varepsilon) \psi^{\prime}(x, \varepsilon)-g(x, \psi(x, \varepsilon), \varepsilon)$ w.r.t. $\varepsilon$ is zero.
} 
Theorem 3. Under the conditions of Theorem 1, and with the notation introduced above, we have:

1. The first nonzero coefficient of $\varepsilon^{1 / m}$ in the expansion of $\mathcal{A}$ is related to the first nonzero coefficient in the expansion of $\left(\zeta_{-}-\zeta_{+}\right)(u, 0, \lambda)$. In fact, they are proportional, and the ratio is precisely $-\rho(\lambda)$, with $\rho(\lambda)$ the nonzero coefficient in (8).

2. The first nonzero coefficient of $\zeta_{-}-\zeta_{+}$can be calculated, either using the previous item if having a pre-existing knowledge of $\mathcal{A}$, or using a Melnikov integral along $\Gamma$ (the heteroclinic connection on the blow up locus from $\zeta_{-}$ to $\left.\zeta_{+}\right)$. The integrands can be obtained through a formal recursive process.

Finally, we present some classes of vector fields having expression (1) for which the assumptions of this paper are satisfied.

For $h=f$ or $g$, we introduce the notation

$$
h_{\mathrm{o}}:=h(0,0,0,0, \lambda), \quad h_{x}:=\frac{\partial h}{\partial x}(0,0,0,0, \lambda),
$$

and similar notation for other partial derivatives.

Theorem 4. If

(i) $f_{\circ}=0, f_{y} \neq 0$ (existence of critical curve by means of implicit function theorem);

(ii) $f_{x}=0, f_{x x} \neq 0$ (at the origin, normal hyperbolicity is lost in the most generic way);

(iii) $g_{0}=0, g_{x} \neq 0$ (connection condition);

(iv) $g_{x}\left|\begin{array}{cc}f_{y} & f_{a} \\ f_{x y} & f_{x a}\end{array}\right|-f_{x x}\left|\begin{array}{cc}f_{y} & f_{a} \\ g_{y} & g_{a}\end{array}\right| \neq 0$ (breaking parameter condition);

(v) the product $g_{x} f_{y}$ is negative (transition from attracting to repelling).

Then, near $(x, y)=(0,0)$ and for $(a, \varepsilon)$ sufficiently small, Assumptions 1-6 are verified for the vector field (1), using the blow up $(x, y, \varepsilon)=\left(u \bar{x}, u^{2} \bar{y}, u^{2} \bar{\varepsilon}\right)$ and writing $a=u A$. Also, the conditions of Theorem 2 are satisfied.

Remark. it may be appropiate to perform some coordinate changes to get the conditions independent of $\lambda$, as required.

The next theorem generalizes the previous one, however, in this case a formal power series solution generally does not exist.

\section{Theorem 5. If}

(i) $f_{0}=0, f_{y} \neq 0$ (existence of critical curve by means of implicit function theorem);

(ii) $f_{x}=0, f_{x x}=0, \ldots, f_{x^{2 n-1}}=0, f_{x^{2 n}} \neq 0$ (at the origin, normal hyperbolicity is lost);

(iii) $g_{0}=0, g_{x}=0, \ldots, g_{x^{2 n-2}}=0, g_{x^{2 n-1}} \neq 0$ (connection condition);

(iv) $g_{x^{2 n-1}}\left|\begin{array}{cc}f_{y} & f_{a} \\ f_{x y} & f_{x a}\end{array}\right|-f_{x^{2 n}}\left|\begin{array}{cc}f_{y} & f_{a} \\ g_{y} & g_{a}\end{array}\right| \neq 0$ (breaking parameter condition);

(v) the product $g_{x^{2 n-1}} f_{y}$ is negative (transition from attracting to repelling).

Then, near $(x, y)=(0,0)$ and for $(a, \varepsilon)$ sufficiently small, Assumptions 1-6 are verified for the vector field (11), using the blow up $(x, y, \varepsilon)=\left(u \bar{x}, u^{2 n} \bar{y}, u^{2 n} \bar{\varepsilon}\right)$ and writing $a=u^{2 n-1} A$. 


\section{Proof of Theorem 1}

3.1. Existence of center manifolds along $\gamma_{ \pm}$. In this section, we will saturate any boundary condition curve $\Sigma_{ \pm}$along the vector field described in Theorem 11. to form a smooth invariant manifold (called center manifold). We will not describe crossing over the contact point here. We will only treat $\Sigma_{-}$; the other case can be handled similarly after reversing time.

Let $L$ be a compact piece of the critical curve $\gamma_{-}$of $X_{\varepsilon, 0, \lambda}$, bounded away from the contact point. In this compact set, $L$ is normally hyperbolic, and at each point of $L$ we can always choose a local $C^{k}$ center manifold $W$ for the vector field $X_{\varepsilon, a, \lambda}$ in $(x, y, \varepsilon, a)$-space (see [K] or [HPS]).

Lemma 3.1. Let $k \in \mathbb{N} \backslash\{0\}$ be fixed. In points of $L$, choosing a $C^{k}$ center manifold for $X_{\varepsilon, a, \lambda}$, the vector field $X_{\varepsilon, a, \lambda}$ is $C^{k}$-equivalent to

$$
\left\{\begin{array}{l}
\dot{x}=-x \\
\dot{y}=g(y, \varepsilon, a, \lambda),
\end{array}\right.
$$

where $g$ is a positive $C^{k}$ function for $\varepsilon \neq 0$ and $g(y, 0, a, \lambda)=0$. The chosen center manifold is mapped to $\{x=0\}, L$ is mapped to $\{x=\varepsilon=a=0\}$ under this equivalence, and the slow dynamics are directed towards the contact point (i.e. increasing $y$ means moving towards the contact point).

Note that often, one can consider that $g(y, \varepsilon, a, \lambda)=\varepsilon^{\sigma}$ for some $\sigma \geq 1$, but in general this is not possible without extra blow ups (think, for example, of a situation where $\left.g(y, \varepsilon, a, \lambda)=\varepsilon\left(\varepsilon^{2}+y^{2}+\lambda^{2}\right)\right)$. Before proving this lemma, let us recall a theorem, that we will not only use to prove Lemma 3.1. but also to prove more complicated statements later on.

Theorem 6 ( $\overline{\mathrm{Bon}})$. Let $X_{\lambda}(x, y, z, a)$ be a $C^{\infty}$ family of vector fields on $\mathbb{R}^{4}$ having the following properties:

(i) $(0,0,0,0)$ is a singular point of $X_{\lambda}$.

(ii) $X_{\lambda}$ is tangent to the foliation $d a=0$.

(iii) $X_{\lambda}$ is tangent to the foliation $d F(y, z)=0$ where $F(y, z)=y^{p} z^{q}$ for $(p, q)=(0,1)$ or $p, q \in \mathbb{N} \backslash\{0\}$ and relatively prime.

(iv) $D X_{\lambda}(0,0,0,0)$ has exactly one nonzero eigenvalue and the related eigenspace is given by $y=z=a=0$.

Let $W$ be a $C^{k}$ center manifold of $X_{\lambda}$ at $(0,0,0,0)$ with $k \in \mathbb{N} \backslash\{0\}$.

Then there exists a local $C^{k}$ change of coordinates $\varphi$ of the form

$$
(x, y, z, a) \mapsto\left(\varphi_{1}(x, y, z, a, \lambda), \varphi_{2}(x, y, z, a, \lambda), \varphi_{3}(x, y, z, a, \lambda), a\right)
$$

with

$$
F\left(\varphi_{2}(x, y, z, a, \lambda), \varphi_{3}(x, y, z, a, \lambda)\right)=F(y, z)
$$

and a strictly positive $C^{k}$ function $h(x, y, z, a)$ such that

$$
\left[h . \varphi_{*} X\right](x, y, z, a)= \pm x \frac{\partial}{\partial x}+Y_{\lambda}(y, z, a),
$$

with $Y_{\lambda}$ of class $C^{k}, Y . a=0, Y . F=0$ and $\varphi(W)=\{x=0\}$.

Proof of Lemma 3.1. We follow the techniques in DR. Take any point of $L$, then a translation will take this point to the origin. Due to the normal hyperbolicity along $L$, a linear change of coordinates will ensure that the linear part of the 
vector field for $\varepsilon=0$ looks like $\left(\begin{array}{cc}0 & 0 \\ 0 & \pm 1\end{array}\right)$. Apply the theorem now: the vector field $\pm x \frac{\partial}{\partial x}+g(y, \varepsilon, a, \lambda) \frac{\partial}{\partial y}$ is $C^{k}$-equivalent to the original one. Due to Assumption 2, $g$ has no zeroes for $\varepsilon>0$ in a neighbourhood of $(y, \varepsilon, a)=(0,0,0)$. By changing $x \rightarrow-x$ and $t \rightarrow-t$ if necessary, we can make $g$ positive, and get to the expression in the lemma.

Proposition 3.2. The saturation of $\Sigma_{-}$is a smooth invariant manifold along any compact piece of the critical curve L, except at the corner point $c_{-}$. This point is the limit of the fast orbit of the end point of $\Sigma_{-}$on the critical curve. At the point $c_{-}$, the saturation of $\Sigma_{-}$is continuous.

Proof. For $\varepsilon \neq 0$ the saturation of $\Sigma_{-}$is clearly $C^{\infty}$, since we only deal with regular $C^{\infty}$ vector fields. The only problem hence deals with the extension for $\varepsilon=0$. It is assumed that the end point of $\Sigma_{-}$lies in the basin of attraction of the critical curve $\gamma_{-}$. This means that in a neighbourhood along the fast orbit of the end point, no singularities appear. Hence, $(\varepsilon, a)$ are regular perturbation parameters in that neighbourhood and the saturation of $\Sigma_{-}$will be smooth. We can extend this manifold until we enter a neighbourhood of $c_{-}$where a normal form can be used. The normal form specified in Lemma 3.1 can be solved implicitly: given a boundary condition curve $\left\{x=\gamma(\varepsilon, \lambda), y=y_{0}\right\}$ the saturation is a graph

$$
x(y, \varepsilon, \lambda)=\gamma(\varepsilon, \lambda) \exp \left(-\int_{y_{0}}^{y} \frac{d s}{g(s, \varepsilon, a, \lambda)}\right)
$$

where $y_{0}$ is the $y$-coordinate of $c_{-}$. We prove the smoothness of this expression as $\varepsilon \rightarrow 0$ in Proposition A.1 (see the appendix). (Note that admissible boundary curve $\gamma$ need not be $C^{k}$ at $\varepsilon \rightarrow 0$, but at least there is an $N \in \mathbb{N}$ so that $\varepsilon^{N} \gamma$ is $C^{k}$ as $\varepsilon \rightarrow 0$; see the definition of admissible boundary curves. This is a slight obstruction, but can be removed by applying Proposition A.7, where it is proved that $\exp \left(-\int_{y_{0}}^{y} \frac{d s}{g(s, \varepsilon, a, \lambda)}\right)$ is $O\left(\varepsilon^{N}\right)$ for all $N$.) Since we can cover the compact $L$ by a finite number of neighbourhoods where a normal form as in Lemma 3.1 is valid, the required smoothness of the saturation of $\Sigma_{-}$along $L$ (except in $c_{-}$) follows.

We recall that $C^{k}$-smoothness for all $k$ is enough in our case to conclude $C^{\infty}$ smoothness, because the domain in which $C^{k}$-smoothness is proved does not shrink as $k$ increases.

3.2. Extending manifolds in the blow up space. To extend the center manifolds from Section 3.1, we need to blow up the origin. For more information regarding family blow up, we refer to the literature. Recall that the parameter plane $(\varepsilon, a)$ might have already been rescaled, as in (9) with $E=1$, so that is why this section uses the vector field (10) with parameters $(v, A)$. In any case, $v$ plays the role of singular parameter, while $A$ is a regular breaking parameter. We will not blow up $A$, nor $\lambda$. Let

$$
\Phi: \mathbb{R}^{+} \times S^{2} \rightarrow \mathbb{R}^{3}:(u,(\bar{x}, \bar{y}, \bar{v})) \mapsto(x, y, v)=\left(u^{p} \bar{x}, u^{q} \bar{y}, u^{m} \bar{v}\right),
$$

where $p, q$ and $m$ are natural numbers representing weights for the variables $x, y$ and $v$. The number $m$ will be especially important, since all objects will be smooth in $u$, and hence smooth in $v^{1 / m}$ - which might be $\varepsilon^{1 / m k}$ if a preliminary scaling $\varepsilon=v^{k}$ has taken place, as in (9) with $E=1$ (see also the remark after Theorem 1). 
The preimage of $\gamma_{-}$is a subset

$$
\bar{\gamma}_{-} \subset\{\bar{v}=0\} .
$$

So the first place to look is in a chart $\bar{v} \sim 0$. In other words, we can look in the chart $(\bar{x}, \bar{y}) \in S^{1}\left(S^{1}\right.$ seen as the circle $\{\bar{v}=0\}$ inside $\left.S^{2}\right)$. Let $(\bar{x}, \bar{y})$ be represented by an angular value $z$ in the neighbourhood of the end point of $\bar{\gamma}_{-}$, and assume $z=0$ corresponds to this end point.

Lemma 3.3. Near $P_{-}$, and for any $k \in \mathbb{N}$, the vector field $\Phi^{*}(X)$ is $C^{k}$ equivalent to

$$
\left\{\begin{aligned}
\dot{u} & =-u \bar{v} h(u, \bar{v}, A, \lambda), \\
\dot{\bar{v}} & =m \bar{v}^{2} h(u, \bar{v}, A, \lambda), \\
\dot{z} & =-z,
\end{aligned}\right.
$$

for some $C^{k}$ function $h$.

Proof. Again, we follow the techniques in [DR, and apply Theorem 6. The necessary conditions can be readily checked: the existence of a $C^{k+2}$-center manifold follows from the general theory in e.g. [HPS; the presence of the foliation is a result of the blow up: $d\left(u^{m} \bar{v}\right)=0$ and we also know $d A=0$, while the partial hyperbolicity is presumed in Assumption 3 (normal hyperbolicity at the end point $\left.P_{-}\right)$.

Hence, a normal form $z \frac{\partial}{\partial z}+Y_{\lambda}(u, \bar{v}, a)$ is obtained, with $Y$ tangent to the foliation $d A=0$ and $d\left(u^{m} \bar{v}\right)=0$. Write

$$
Y_{\lambda}(u, \bar{v}, a)=h_{1}(u, \bar{v}, A, \lambda) \frac{\partial}{\partial u}+h_{2}(u, \bar{v}, A, \lambda) \frac{\partial}{\partial \bar{v}} .
$$

The set $\{u \bar{v}=0\}$ is preserved under the normal form with $\{u=0\}$ as part of the blow up locus, and $\{\bar{v}=0\}$ outside the blow up locus (corresponding to the manifold $\{\varepsilon=0\}$ ). In any case, both spaces are invariant under $Y_{\lambda}$. This invariance implies that $h_{1}=O(u)$, and hence

$$
h_{1}(u, \bar{v}, A, \lambda)=-u h_{3}(u, \bar{v}, A, \lambda),
$$

for some function $h_{3}$. The tangency to $d\left(u^{m} \bar{v}\right)=0$ then yields

$$
h_{2}(u, \bar{v}, A, \lambda)=m \bar{v} h_{3}(u, \bar{v}, A, \lambda) .
$$

The center manifold $\{z=0\}$ has to include a line of singularities $\{z=0, \bar{v}=0\}$. We conclude that $h_{3}=O(v)$ and

$$
h_{3}(u, \bar{v}, A, \lambda)=\bar{v} h(u, \bar{v}, A, \lambda)
$$

for some function $h$. Since we started with a $C^{k+2}$ normal form, $h$ will at least be $C^{k}$.

The existence of $C^{k}$ normal forms for any $k$ enables us to prove $C^{\infty}$ smoothness of invariant manifolds:

Proposition 3.4. The saturation of $\Sigma_{-}$forms a smooth invariant manifold in the blow up space, in a neighbourhood of the corner point $P_{-}$.

Proof. We use the normal form in Lemma 3.3 to prove the $C^{k}$-smoothness. We may assume that $\Sigma_{-}$is inside the neighbourhood where the normal form is valid, using Proposition 3.2 if necessary. We may suppose that the curve is a graph 
$\left\{z=\gamma(\bar{v}), u=u_{0}>0\right\}$, and we suppose that $u_{0}>0$ is chosen such that all $(u, v) \in\left[0, u_{0}\right]^{2}$ are in the definition domain of the function $h$ of Lemma 3.3.

We can reduce to the case $m=1$ by writing $\tilde{u}=u^{m}$ :

$$
\left\{\begin{aligned}
\dot{\tilde{u}} & =-\tilde{u} \bar{v} \tilde{h}(\tilde{u}, \bar{v}, A, \lambda) \\
\dot{\bar{v}} & =\bar{v}^{2} \tilde{h}(\tilde{u}, \bar{v}, A, \lambda) \\
\dot{z} & =-z
\end{aligned}\right.
$$

with

$$
\tilde{h}(\tilde{u}, \bar{v}, A, \lambda):=m h\left(\tilde{u}^{1 / m}, \bar{v}, A, \lambda\right) .
$$

It seems that we loose differentiability in terms of $\tilde{u}$, but for the remainder of the proof, we just need that $\tilde{u}^{k} \tilde{h}$ is $C^{k}$, and this is still the case.

So assume now $m=1$ (and drop the tildes). Observe that Assumption 4 implies that $h$ is nonzero for $\bar{v}>0$, and from Assumption 2 we know that its sign is positive. So, in the domain $u \bar{v}>0$, (15) is equivalent to

$$
\left\{\begin{aligned}
\dot{u} & =-u \bar{v} \\
\dot{\bar{v}} & =\bar{v}^{2} \\
\dot{z} & =-z / h(u, \bar{v}, A, \lambda) .
\end{aligned}\right.
$$

Fixing $\left(u_{1}, \bar{v}_{1}\right)$ the orbit in negative time of $\left(u_{1}, \bar{v}_{1}, z_{1}\right)$, for any $z_{1}$, crosses the plane $u=u_{0}$ at $\bar{v}=u_{1} \bar{v}_{1} / u_{0}$. So, if we take $\left(u_{0}, u_{1} \bar{v}_{1} / u_{0}, \gamma\left(u_{1} \bar{v}_{1} / u_{0}\right)\right)$ as initial conditions for $(u, \bar{v}, z)$, and if we follow the orbit for a time $T\left(u_{1}, \bar{v}_{1}\right)=\frac{u_{0}-u_{1}}{u_{1} \bar{v}_{1}}$, then we reach a point $\left(u_{1}, \bar{v}_{1}\right)$ of the saturation of the chosen curve. This yields a graph $z=z\left(u_{1}, \bar{v}_{1}\right)$ :

$$
z\left(u_{1}, \bar{v}_{1}\right)=\gamma\left(u_{1} \bar{v}_{1} / u_{0}\right) \exp \left(-\int_{0}^{T\left(u_{1}, \bar{v}_{1}\right)} \frac{d t}{h\left(u_{0}-u_{1} \bar{v}_{1} t, \frac{u_{1} \bar{v}_{1}}{u_{0}-u_{1} \bar{v}_{1} t}, A, \lambda\right)}\right) .
$$

Writing

$$
t=T\left(u_{1}, \bar{v}_{1}\right) s,
$$

the above expression yields

$$
z\left(u_{1}, \bar{v}_{1}\right)=\gamma\left(u_{1} \bar{v}_{1} / u_{0}\right) \exp \left(-\int_{0}^{1} \frac{d s}{g\left(s, u_{1}, \bar{v}_{1}, A, \lambda\right)}\right)
$$

with

$$
g\left(s, u_{1}, \bar{v}_{1}, A, \lambda\right):=\frac{u_{1} \bar{v}_{1}}{u_{0}-u_{1}} h\left(u_{0}-\left(u_{0}-u_{1}\right) s, \frac{u_{1} \bar{v}_{1}}{u_{0}-\left(u_{0}-u_{1}\right) s}, A, \lambda\right) .
$$

For $u_{1}$ sufficiently small (let us say $u_{1}<\frac{1}{2} u_{0}$ ), and $0 \leq s<1$ the function $g$ is $C^{k}$ if $u_{1} \bar{v}_{1} \geq 0$ and positive if $u_{1} \bar{v}_{1}>0$. A problem could arise at $s=1$; let us, however, remark that, in the region under consideration (including $s=1$ )

$$
0<\frac{u_{1}}{u_{0}-\left(u_{0}-u_{1}\right) s} \leq 1,
$$

while

$$
\frac{\partial^{n}}{\partial u_{1}^{n}}\left(\frac{u_{1}}{u_{0}-\left(u_{0}-u_{1}\right) s}\right)=(-1)^{n-1} n ! \frac{u_{0} s^{n-1}(1-s)}{\left(u_{0}-\left(u_{0}-u_{1}\right) s\right)^{n+1}},
$$

such that $u_{1}^{n} \frac{\partial^{n}}{\partial u_{1}^{n}}\left(\frac{u_{1}}{u_{0}-\left(u_{0}-u_{1}\right) s}\right)$ is bounded. It hence easily follows that

$$
u_{1}^{k-1} g\left(s, u_{1}, \bar{v}_{1}, A, \lambda\right)
$$


is of class $C^{k}$. This observation will allow us to use Proposition A.7 in the appendix. From this proposition it follows that

$$
\exp \left(-\int_{0}^{S} \frac{d s}{g\left(s, u_{1}, \bar{v}_{1}, A, \lambda\right)}\right)
$$

is $C^{k}$ for all $0<S \leq 1, u_{1} \bar{v}_{1} \geq 0$. Intersecting with the plane $S=1$ gives us the smoothness of $z\left(u_{1}, \bar{v}_{1}\right)$, and we also see that $z\left(u_{1}, \bar{v}_{1}\right)$ is $C^{k}$-flat at $u_{1} \bar{v}_{1}=0$.

We have shown that the saturation is $C^{k}$ in all points including the boundary $u_{1} \bar{v}_{1}=0$. The boundary consists of the critical curve up to its intersection with the blow up locus, together with the invariant manifold on the blow up locus. Once we have passed the corner point, no more singularities are expected in a neighbourhood of $A=0, u=0$ (Assumption 4). This means that we can saturate the curve beyond the neighbourhood where the normal form is valid. This implies that the saturation is $C^{k}$ in a neighbourhood that does not depend on $k$. Since the result holds for all $k$, this proves the $C^{\infty}$ smoothness in a neighbourhood of $P_{-}$.

3.3. Connecting the center manifolds. The saturation of the section $\Sigma_{-}$forms a smooth invariant manifold $W_{-}$, as above, and by reversing time, so will the saturation of $\Sigma_{+}$along $-X_{v, A, \lambda}$. In this work, we had to reduce the neighbourhood in which $v$ can vary, possibly it has been necessary to restrict $A$ to a small neighbourhood of the origin; we did not put constraints on the compact set $\Lambda$.

To connect the two manifolds $W_{-}$and $W_{+}$together (and hence construct canard solutions), we consider a chart on the blow up locus where both manifolds are shown to exist. This is the family rescaling chart, shortly denoted as the chart $\bar{v}=1$. Here, the variables are rescaled as follows:

$$
\left\{\begin{array}{l}
x=u^{p} \bar{x} \\
y=u^{q} \\
v=u^{m}
\end{array}\right.
$$

In this chart, $u$ becomes a regular perturbation parameter; we obtain a family of vector fields

$$
\bar{X}_{u, a, \lambda}:\left\{\begin{aligned}
\dot{\bar{x}} & =\bar{f}(\bar{x}, \bar{y}, u, A, \lambda) \\
\dot{\bar{y}} & =\bar{g}(\bar{x}, \bar{y}, u, A, \lambda) .
\end{aligned}\right.
$$

We have assumed in Assumption 5 (connection condition) that a heteroclinic connection $\Gamma$ exists that connects $\gamma_{-}$to $\gamma_{+}$. Since $\bar{X}$ does not have any singularities in the neighbourhood of any compact piece of $\Gamma$, we can extend the two manifolds $W_{-}$and $W_{+}$to meet in a transversal section $T$ : choose a smooth transverse section (transversally intersecting $\Gamma$, and hence locally transverse to the flow of $\bar{X}$ ), then $W_{-}$intersects $T$ in a smooth curve inside $T$, and so will $W_{+}$. Denote these curves by $\zeta_{-}$and $\zeta_{+}$.

Let $\sigma$ be the intersection of $T$ with the blow up locus $\{u=0\}$. Choose a coordinate system on $\sigma$, and denote the coordinate by $z$. Then, locally $(z, u)$ is a coordinate system in $T$, and $\zeta_{-}$and $\zeta_{+}$are graphs in $(u, A, \lambda)$. Let $z_{ \pm}(u, A, \lambda)$ be such graph representations (w.r.t. some fixed coordinate system of $T$ ), then from Assumptions 5 and 6 we know:

(i) $z_{-}(0,0, \lambda)=z_{+}(0,0, \lambda)$,

(ii) $\frac{\partial z_{-}}{\partial A}(0,0, \lambda) \neq \frac{\partial z_{+}}{\partial A}(0,0, \lambda)$. 
The first condition is merely the existence of a heteroclinic connection $\Gamma$ on the blow up locus for $A=0$. In Proposition 5.7 we derive a formula that could be used to check the above equations.

Thus, under these assumptions, we can use the implicit function theorem to solve

$$
z_{-}(u, \mathcal{A}(u, \lambda), \lambda)=z_{+}(u, \mathcal{A}(u, \lambda), \lambda)
$$

for $\mathcal{A}$. This proves Theorem 1 .

\section{Proof of Theorem 2}

The smoothness of manifolds in the blow up space does not necessarily imply the smoothness in the standard phase space. But, we can recover a great deal. First, outside the blow up locus the blow up map is a regular diffeomorphism, so outside the origin we can prove the smoothness of the constructed invariant manifolds. To show smoothness at the origin, extra arguments are needed.

A second observation is the necessity of the conditions in Theorem 2 If a formal expansion does not exist at the origin, then the manifolds can never be smooth there - this is because the Taylor expansion of any smooth manifold would agree with these conditions.

A third observation is relevant: sometimes, a formal expansion can be found to be formally invariant under the vector field, up to order $k$. In that case, we are able to prove the $C^{k}$-smoothness of the invariant manifolds in the origin.

4.1. Reduction to $(v, A)$ parameters. The formal expansions in Theorem 2 are expressed in terms of $(\varepsilon, a)$, but if necessary, we can replace the expansions by expansions in terms of $(v, A)$. Indeed, if

$$
(a, \varepsilon)=\left(v^{k} A, v^{\ell}\right),
$$

for some $(k, \ell)$ (following the rescaling in (91)), we can translate the formal power series $\hat{a}$ to a series in $\hat{A}$; this yields $\hat{A}=v^{-k} \hat{a}\left(v^{\ell}, \lambda\right)$. In this section we will focus on two results: on one hand, that $\hat{A}$ is a genuine power series in $v$ and on the other hand, that smoothness in terms of $(v, \lambda)$ of canard manifolds is equivalent to smoothness in terms of $(\varepsilon, \lambda)$.

Instead of continuing to work with the formal power series, we realize these series as Taylor series of smooth functions; this is possible due to Borel's theorem (see e.g. [WA]). Choose

$$
a=\tilde{a}(\varepsilon, \lambda), \quad y=\tilde{\phi}(x, \varepsilon, \lambda) .
$$

We can consider $y=\tilde{\phi}(x, \varepsilon, \lambda)$ as a perturbation of a canard manifold $W$, but it is advantageous to see it as a canard manifold of a perturbed vector field. We therefore introduce an error function for $(\tilde{\phi}, \tilde{A})$ :

$$
E(x, \varepsilon, \lambda):=f(x, \tilde{\phi}(x, \varepsilon), \varepsilon, \tilde{a}(\varepsilon, \lambda), \lambda) \frac{\partial \tilde{\phi}}{\partial x}(x, \varepsilon)-v g(x, \tilde{\phi}(x, \varepsilon), \varepsilon, \tilde{a}(\varepsilon, \lambda), \lambda) .
$$

Due to the formal invariance of $(\hat{a}, \hat{\phi})$, we know that $E$ is flat in $\varepsilon$, uniformly in $(x, \lambda)$. Consider now a slightly altered vector field

$$
\tilde{X}_{\varepsilon, a, \lambda}:\left\{\begin{aligned}
\dot{x} & =f(x, y, \varepsilon, a, \lambda) \\
\dot{y} & =v g(x, y, \varepsilon, a, \lambda)+E(x, \varepsilon, \lambda) .
\end{aligned}\right.
$$

Clearly, for the family $\tilde{X}$ the same blow up procedure as for $X$ leads to a good desingularization. Hence, Theorem 1 applies to $\tilde{X}$ and we can choose a control 
curve $a=\mathcal{A}(\varepsilon, \lambda)$ as a blow down of a $C^{\infty}$ control curve expressed in $(A, v)$ coordinates. Because the same blow up weights were used, it is clear that $\mathcal{A}(\varepsilon, \lambda)=$ $\varepsilon^{k / \ell} \mathcal{A}_{1}\left(\varepsilon^{1 / \ell}, \lambda\right)$. Because $\mathcal{A}$ is infinitely tangent to $\tilde{a}$, it is clear that $\hat{A}=\hat{\mathcal{A}}_{1}(v, \lambda)$, implying that it is a genuine series in $v$.

We can now safely state that under the conditions of Theorem 2 there exist

$$
\hat{A}=\sum_{n=0}^{\infty} A_{n}(\lambda) v^{n}, \quad \hat{\tilde{y}}=\sum_{n=0}^{\infty} \tilde{y}_{n}(x, \lambda) v^{n},
$$

with $\hat{\tilde{y}}$ being a formal canard "solution" of (10), and where $\tilde{y}$ is obtained using $\hat{A}=v^{-k} \hat{a}$. Assume for a moment that we can prove that the graph $y=\tilde{\Psi}(x, v, \lambda)$ is smooth in terms of $(x, v, \lambda)$, then we can go back to the original parameters:

$$
y=\Psi(x, \varepsilon, \lambda):=\tilde{\Psi}\left(x, \varepsilon^{1 / \ell}, \lambda\right)
$$

and because the formal expansion of $\Psi$ only has terms in powers of $v^{\ell}$, the above function will still be smooth in $\varepsilon$. Similarly, the smoothness of a control curve in terms of $v$ will imply the smoothness of the control curve in terms of $\varepsilon$.

4.2. Canard solution manifold as a graph. Suppose a formal expansion as in Theorem 2 exists. From now on, we will interpret Theorem 2 in terms of $(v, A)$ instead of in terms of $(\varepsilon, a)$. Reading this section, one can treat $(v, A)$ as being equal to $(\varepsilon, a)$, but for the sake of generality we prefer to make a distinction.

Take a control curve $\mathcal{A}(v, \lambda)$ and a manifold of canard solutions $W$ as in Theorem 1. The blow down of this manifold $W$ is, when restricted to $v=0$, at least continuous at the origin (because of the existence of a connection on the blow up locus). Outside the origin, this restriction must coincide with the critical curve, and this critical curve can be written as a graph in $x$ (this is assumed in Theorem 2). Hence, locally around the origin, we can blow down $W$ to a graph in $(x, v, \lambda)$. Assume now that

$$
y=\Psi(x, v, \lambda)
$$

is the blow down of $W$. We already know that it is smooth outside $(x, v)=(0,0)$, for $x$ close to 0 . To prove Theorem 2, it suffices now to prove:

Proposition 4.1. For all $n, r \in \mathbb{N}$ we have

$$
\lim _{v \rightarrow 0} \frac{1}{n !} \frac{\partial^{n+r} \Psi}{\partial v^{n} \partial x^{r}}(x, v, \lambda)=\frac{\partial^{r} y_{n}}{\partial x^{r}}(x, \lambda),
$$

where $y_{n}$ is defined in the statement of Theorem 2.

As before we realize these series as Taylor series of smooth functions

$$
A=\tilde{A}(v, \lambda), \quad y=\tilde{\phi}(x, v, \lambda),
$$

so that the infinite jet of $\tilde{A}$ resp. $\tilde{\phi}$ coincides with the formal power series $\hat{A}$ resp. $\hat{\phi}$. In view of proving the proposition, we can now say that it is necessary to prove:

$$
\left\{\begin{array}{l}
\lim _{v \rightarrow 0} \frac{\partial^{n+r} \Psi}{\partial v^{n} \partial x^{r}}(x, v, \lambda)=\frac{\partial^{n+r} \tilde{\phi}}{\partial v^{n} \partial x^{r}}(x, 0, \lambda), \\
\lim _{v \rightarrow 0} \frac{\partial^{n} \mathcal{A}}{\partial v^{n}}(v, \lambda)=\frac{\partial^{n} \tilde{A}}{\partial v^{n}}(0, \lambda),
\end{array}\right.
$$


uniformly in $\lambda$ and for all $(n, r)$. One can look at these expressions in blow up coordinates, in the various charts. In the family rescaling chart $(\bar{v}=1)$ it suffices to check that

$$
\left\{\begin{array}{l}
\lim _{u \rightarrow 0} \frac{\Psi\left(u^{p} \bar{x}, u^{m}, \lambda\right)-\tilde{\phi}\left(u^{p} \bar{x}, u^{m}, \lambda\right)}{u^{s}}=0 \\
\lim _{u \rightarrow 0} \frac{\mathcal{A}\left(u^{m}, \lambda\right)-\tilde{A}\left(u^{m}, \lambda\right)}{u^{s}}=0
\end{array}\right.
$$

uniformly in $\lambda$ and for all $s \in \mathbb{N}$. We do not need to prove similar conditions on $\frac{\partial^{n+r} \Delta}{\partial v^{n} \partial x^{r}}$ with $\Delta=\Psi-\tilde{\phi}$, since the function $\bar{\Delta}(u, \bar{x}, \lambda):=\Delta\left(u^{p} \bar{x}, u^{m}, \lambda\right)$ is a $C^{\infty}$ function. The existing relations between the derivatives w.r.t. $(v, x)$ and the derivatives of $\bar{H}$ w.r.t. $(u, \bar{x})$ will imply the necessary conditions.

We must investigate these expressions in a full neighbourhood of $(v, x)=(0,0)$, so it is necessary to look at the phase directional rescaling as well. There, the denominator will be of the form $\left(u^{r} \bar{v}^{\ell}\right)$ for arbitrary $(r, \ell)$. Assuming that one needs to look in the directional chart $\{\bar{x}=-1\}$, one finds sufficient conditions to be

$$
\lim _{u^{m} \bar{v} \rightarrow 0} \frac{\Psi\left(-u^{p}, u^{m} \bar{v}, \lambda\right)-\tilde{\phi}\left(-u^{p}, u^{m} \bar{v}, \lambda\right)}{u^{r} \bar{v}^{s}}=0 .
$$

(If the above equation is true, then one has that the numerator is $O\left(u^{r} \bar{v}^{s}\right)$, and, due to the smoothness in terms of $(u, \bar{v})$, that its $n$th derivative w.r.t. $u$ is $O\left(u^{r-n} \bar{v}^{s}\right)$. Similarly, derivatives w.r.t. $\bar{v}$ and $\lambda$ are treated.)

4.3. Perturbing the vector field. We can consider $y=\tilde{\phi}$ as a perturbation of the manifold $W$, but we can also regard it as a manifold of canard solution of a perturbed vector field. We therefore introduce an error function for $(\tilde{\phi}, \tilde{A})$ :

$$
E(x, v, \lambda):=f(x, \tilde{\phi}(x, v), v, \tilde{A}(v, \lambda), \lambda) \frac{\partial \tilde{\phi}}{\partial x}(x, v)-v g(x, \tilde{\phi}(x, v), v, \tilde{A}(v, \lambda), \lambda) .
$$

Due to the formal invariance of $\hat{A}$ and $\hat{y}$, we know that $E$ is flat in $v$, uniformly in $(x, \lambda)$. Consider now a slightly altered vector field

$$
\tilde{X}_{v, A, \lambda}:\left\{\begin{aligned}
\dot{x} & =f(x, y, v, A, \lambda) \\
\dot{y} & =v g(x, y, v, A, \lambda)+E(x, v, \lambda) .
\end{aligned}\right.
$$

Then, the graph $y=\tilde{\phi}(x, v, \lambda)$ defines a smooth manifold $\tilde{W}$ and $(\tilde{A}, \tilde{W})$ is a manifold of canard solutions for $\tilde{X}$.

So, on one hand, we have a family of vector fields (10), and on the other hand, we have a family of vector fields (19) from which we know that it has an invariant manifold $\{A=\tilde{A}(v, \lambda), y=\tilde{\phi}(x, v, \lambda)\}$. Both families of vector fields are strongly related to each other: the infinite jets with respect to $v$ of both vector fields are the same.

Consider the blow up map introduced for the vector field $X$. If we apply the same blow up map on $\tilde{X}$, then we can compare $X$ with $\tilde{X}$ in blow up coordinates. They are everywhere infinitely tangent to each other along the blow up locus. Looking in the phase directional rescaling charts, we used $C^{k}$-normal form coordinates as in Lemma 3.3 so applying the same transformation to $\overline{\tilde{X}}$, it is not hard to prove 
that the perturbed vector field is

$$
\overline{\tilde{X}}:\left\{\begin{aligned}
\dot{u} & =-u \bar{v} \tilde{h}(u, \bar{v}, z, A, \lambda) \\
\dot{\bar{v}} & =m \bar{v}^{2} \tilde{h}(u, \bar{v}, z, A, \lambda) \\
\dot{z} & =-z+\tilde{f}(u, \bar{v}, z, A, \lambda)
\end{aligned}\right.
$$

where $\tilde{h}-h=O\left(u^{r} \bar{v}^{\ell}\right), \tilde{f}=O\left(u^{r} \bar{v}^{\ell}\right)$ for $(r, \ell)$ arbitrarily high (provided that we choose a $C^{k}$ normal form with $k \geq r+\ell$ and shrink the neighbourhood of $(u, \bar{v}, z)=(0,0,0))$.

The following lemma proves (18):

Lemma 4.2. Let $z=\tilde{\psi}(u, \bar{v}, A, \lambda)$ be invariant under the $C^{k}$ vector field (20) in a neighbourhood of $(u, \bar{v}, z)=(0,0,0)$. Then $z=\tilde{\psi}(u, \bar{v}, A, \lambda)$ is $O\left(u^{r} \bar{v}^{\ell}\right)$ flat to $z=0$, with $r+\ell \leq k$. This asymptotic property is uniform in $\lambda \in \Lambda$ and in $A$ near the origin.

Before proving this lemma, we show how this lemma can be used to prove (17), finishing the proof of Theorem 2. In the family rescaling chart, these invariant manifolds can be extended until they intersect the section $T$, and we can do the same thing in backward time for manifolds coming from the other side. The lemma states that at some point (near infinity in the family rescaling chart) the invariant manifold of $\overline{\tilde{X}}$ is $O\left(u^{r}\right)$-close to the invariant manifold of $\bar{X}$.

Following the flow of $\bar{X}$ in the family rescaling map will not decrease the order of separation between the two manifolds; this is due to the absence of singularities in a tube around the heteroclinic connection $\Gamma$, and due to the fact that $\dot{u}=0$ there.

If we apply the implicit function theorem to connect the center manifolds coming from the left and from the right, then no difference is seen between the perturbed invariant manifolds and the actual invariant manifolds up to order $r$. So in the implicit solution we will see no difference between $\mathcal{A}$ and $\tilde{A}$ up to order $r$. This means that $\mathcal{A}$ and $\tilde{A}$ are asymptotic of order $O\left(u^{r}\right)$ for any $r$. Hence, the second part of (17) is shown. The first part easily follows now too, since from now on, we can treat $\mathcal{A}$ and $\tilde{A}$ to be the same, and the invariant manifold will then stay $O\left(u^{r}\right)$-close to the perturbed invariant manifold uniformly in any compact subset of the family rescaling chart.

It remains to prove the lemma.

Proof of Lemma 4.2. The smoothness of such manifolds has already been proved. So let us first consider the restriction to $u=0$. This restriction is invariant under

$$
\bar{v}^{2} h(0, \bar{v}, A, \lambda) \frac{\partial}{\partial \bar{v}}+(-z) \frac{\partial}{\partial z} .
$$

In the domain $\bar{v} \geq 0$, there is a unique invariant (center) manifold, $z=0$. Hence $\tilde{\psi}=O(u)$. Writing now $z=u z_{1}$, we can pull back the vector field and write it in terms of $\left(u, \bar{v}, z_{1}\right)$. This yields

$$
\left\{\begin{aligned}
\dot{u} & =-u \bar{v} \tilde{h}\left(u, \bar{v}, u z_{1}, A, \lambda\right) \\
\dot{\bar{v}} & =m \bar{v}^{2} \tilde{h}\left(u, \bar{v}, u z_{1}, A, \lambda\right) \\
\dot{z}_{1} & =-\left(1-\bar{v} \tilde{h}\left(u, \bar{v}, u z_{1}, A, \lambda\right)\right) z_{1}+\frac{1}{u} \tilde{f}\left(u, \bar{v}, u z_{1}, A, \lambda\right)
\end{aligned}\right.
$$


which leads to an equivalent vector field

$$
\left\{\begin{aligned}
\dot{u} & =-u \bar{v} \tilde{h}_{1}(u, \bar{v}, z, A, \lambda), \\
\dot{\bar{v}} & =m \bar{v}^{2} \tilde{h}_{1}(u, \bar{v}, z, A, \lambda), \\
\dot{z}_{1} & =-z_{1}+\tilde{f}_{1}\left(u, \bar{v}, z_{1}, A, \lambda\right),
\end{aligned}\right.
$$

but where $\tilde{f}_{1}=O\left(u^{r-1} \bar{v}^{\ell}\right)$ and is a $C^{k-1}$ function. This new vector field has an invariant manifold $z_{1}=\psi_{1}(u, \bar{v}, A, \lambda):=\frac{1}{u} \tilde{\psi}(u, \bar{v}, A, \lambda)$. It remains to prove that $\psi_{1}$ is $O\left(u^{r-1} \bar{v}^{\ell}\right)$. Continuing this process reduces to the case $r=0$. So let us now assume $r=0$. Looking at $\bar{v}=0$, we find that $z_{r}=\tilde{\psi}_{r}(u, 0, A, \lambda)$ is identically 0 , since $\tilde{f}$ is $O\left(\bar{v}^{\ell}\right)$, with $\ell \geq 1$. Hence, we can proceed as before by writing $z=\bar{v} z_{1}$.

\section{Proof of Theorem 3}

In Section 3, we have proved the existence of the "center manifolds" coming from both sides of the blow up locus, and meeting somewhere on the blow up locus. In fact, they only meet for $\varepsilon=a=0$. So what happens when $\varepsilon \neq 0$, or $a \neq 0$ ? In these cases, the manifolds are separated. We derive formulas for calculating the separation of these manifolds.

Since the comparison between the left and right center manifolds is done in the family rescaling charts, we will work most of the time in this chart.

In Section 5.2 we state some facts on saddle connections in the plane using Melnikov theory, which we will use to prove the second part of Theorem 3 .

5.1. The relation between angle and the control curve. We focus on the first part of Theorem 3 . We use the notation from Theorem 3 , Let $\mathcal{A}(u, \lambda)$ be a control curve as in Theorem 1 and define

$$
\Delta(u, A, \lambda)=\zeta_{-}(u, A, \lambda)-\zeta_{+}(u, A, \lambda)
$$

where $\zeta_{ \pm}$hase been defined in (12). $\Delta$ is the separation of the forward and backward center manifolds. Of course, $\Delta$ as well as $\mathcal{A}$ depend on the boundary curves $\Sigma_{-}$ and $\Sigma_{+}$chosen in Theorem 3, but the asymptotic expansion is unique. Suppose

$$
\mathcal{A}(u, \lambda)=a_{r}(\lambda) u^{r}+o\left(u^{r}\right),
$$

for $r \geq 1$ and with $a_{r}(\lambda) \neq 0$; then

$$
\Delta(u, \mathcal{A}(u, \lambda), \lambda) \equiv 0 .
$$

This has an effect on the asymptotic expansion

$$
\Delta(u, 0, \lambda)+\mathcal{A}(u, \lambda) \frac{\partial \Delta}{\partial a}(u, 0, \lambda)=o(\mathcal{A}(u, \lambda)) .
$$

Hence,

$$
\lim _{u \rightarrow 0}\left(\frac{\Delta(u, 0, \lambda)}{\mathcal{A}(u, \lambda)}+\frac{\partial \Delta}{\partial a}(0,0, \lambda)\right)=0 .
$$

Notice that $\frac{\partial \Delta}{\partial a}(0,0, \lambda)$ is the nonzero number $\rho(\lambda)$ in (8) . (An expression for this number is calculated in Proposition 5.7) Conclusion:

$$
\left\{\begin{aligned}
\mathcal{A}(u, \lambda) & =a_{r}(\lambda) u^{r}+o\left(u^{r}\right), \\
\Delta(u, 0, \lambda) & =b_{r}(\lambda) u^{r}+o\left(u^{r}\right),
\end{aligned} \quad \text { with } \frac{b_{r}(\lambda)}{a_{r}(\lambda)}=-\rho(\lambda) \neq 0 .\right.
$$


Note that (21) is slightly more general than (22), since it can also be used in the case $\mathcal{A}(u, \lambda)$ and is infinitely flat in $u$. But in such a case, we must add the assumption that $\mathcal{A}(u, \lambda) \neq 0$ for $u \neq 0$.

5.2. Perturbations of regular orbits in the plane. We intend to study the breaking of a heteroclinic connection on the plane, inducing expressions that can be used in the specific problem that we want to investigate. We will consider a 1-dimensional parameter $\mu$, which will be the bifurcation parameter. The whole setting may depend on other parameters $\lambda$, which are "trivial" in the sense that they do not induce bifurcations. We hence will work with a family, depending on $\lambda$, of 3-dimensional situations. We will keep $\mu$ a 1-dimensional parameter although it is not necessary.

Consider a vector field

$$
X_{\mu}:\left\{\begin{aligned}
\dot{x} & =f(x, y, \mu), \\
\dot{y} & =g(x, y, \mu),
\end{aligned}\right.
$$

where $f$ and $g$ are smooth on $\mathbb{R}^{2} \times P$. We will work most of the time with the extended vector field $X_{\mu}+0 \frac{\partial}{\partial \mu}$. Let $\phi(t,(x, y, \mu))$ be the flow for this vector field. Choose now a (fibred) section $T \subset \mathbb{R}^{2} \times P$ transverse to the flow of $X_{\mu}$, with a coordinate mapping

$$
\psi:(h, \mu) \mapsto\left(\psi_{0}(h, \mu), \mu\right) .
$$

We try to calculate intersections of heteroclinic connections passing through $T$ in these coordinates. In this section, however, instead of a heteroclinic connection, a perturbation of a regular orbit is considered. In the next section, we will see how the results can be maintained if we let the orbit tend to a heteroclinic connection.

Consider the projection on $T$ along the orbits of $X_{\mu}$ :

$$
P(x, y, \mu):=\phi(\tau(x, y, \mu),(x, y, \mu)),
$$

where $\tau$ is the transition time to go from $(x, y, \mu)$ to a point in $T$. Of course, $P$ is not defined everywhere, but is certainly defined in an open neighbourhood of the chosen orbit. One sees that $P$ is constant along orbits of $X_{\mu}$ and hence

$$
H(x, y, \mu):=\left\langle(1,0), \psi^{-1}(P(x, y, \mu))\right\rangle
$$

(the $h$ coordinate of $\left.\psi^{-1}(P(x, y, \mu))\right)$ is a first integral for $X_{\mu}$ in some open neighbourhood of the orbit.

Lemma 5.1. There is an integrating factor $\theta$ defined on the domain of $P$ so that

$$
X_{\mu}:\left\{\begin{array}{l}
\dot{x}=-\theta(x, y, \mu)^{-1} \frac{\partial H}{\partial y}(x, y, \mu), \\
\dot{y}=\theta(x, y, \mu)^{-1} \frac{\partial H}{\partial x}(x, y, \mu) .
\end{array}\right.
$$

Proof. Because $H$ is invariant along orbits of $X_{\mu}$, one has $f \frac{\partial H}{\partial x}+g \frac{\partial H}{\partial y}=0$. So one could define $\theta^{-1}$ as $g / \frac{\partial H}{\partial x}$ in points where the denominator is nonzero, or $-f / \frac{\partial H}{\partial y}$ in other points. Both denominators cannot be zero at the same time (because there are no fixed points).

The key to finding the angle between the manifolds lies in the study of $\theta$. But first, we will show how to use the lemma to calculate the intersection.

Let $\gamma$ be the chosen orbit for $\mu=0$. We study perturbations of $\gamma$ as follows: we consider a vertical line segment (segments of the form $\{(x, y, \mu) \mid \mu \in(\mathbb{R}, 0)\}$, with $(x, y)$ chosen on $\gamma$ ) and let points of such a line segment flow. It is in a 
way unnatural to choose line pieces instead of more general curves, but in view of proving the results in this paper, vertical line segments suffice. The flow of this line segment is intersected with $T$, and compared to $\gamma \cap T$.

The curve $\gamma$ intersects $T$ in a single point, but if we lift the curve vertically $\left(\pi^{-1}(\gamma)\right.$ if $\pi$ is the projection onto $\left.\{\mu=0\}\right)$, then the intersection of $\pi^{-1}(\gamma)$ with $T$ is some curve, say parametrized as $h=c(\mu)$. Any point above $\gamma$ meets this curve, if it follows the flow of $X_{0}$. So consider a point $(p, \mu)$ above $\gamma$ (i.e. $p \in \gamma$ ), and let it flow along $X_{0}$ until it meets $T$. The fundamental theorem of calculus states

$$
c(\mu)-H(p, \mu)=\int X_{0}(H),
$$

where the integration is along the curve $\gamma$ (lifted to height $\mu$ ) from $(p, \mu)$ to $T$. We could calculate $c(\mu)$, but this is not necessary: let $(q, \mu)$ be another point above $\gamma$ at the same height, then

$$
H(p, \mu)-H(q, \mu)=-\int_{\gamma(p, q)} X_{0}(H),
$$

where $\gamma(p, q)$ is the piece of $\gamma$ from $p$ to $q$.

In this section, we will only describe $j_{k}^{\mu} H(p)-j_{k}^{\mu} H(q)$ (writing $j_{k}^{\mu}$ for the $k$-jet w.r.t. $\mu$ ); only in a later section we will proceed to the limit (letting $p$ and $q$ tend to infinity, or in other words, letting $\gamma(p, q)$ tend to a heteroclinic connection).

For fixed $p$ and $q$, we have

$$
j_{k}^{\mu} H(p)-j_{k}^{\mu} H(q)=-\int_{\gamma(p, q)} j_{k}^{\mu} X_{0}(H),
$$

Elaborating the integrand yields

$$
X_{0}(H)=f_{0} \frac{\partial H}{\partial x}+g_{0} \frac{\partial H}{\partial y}=\theta\left(f_{0} g-g_{0} f\right) .
$$

So, if we are able to calculate $\theta$ up to any order, then we can calculate the contact between the invariant manifolds up to any order.

For calculating $\theta$ up to any order, observe that manipulating the two forms for $X_{\mu}$ will show that

$$
X_{\mu}(\theta)=-\theta \operatorname{div} X_{\mu}
$$

This expression gives $\theta$ as a solution to a differential equation; if we have an initial condition for $\theta$, we can calculate $\theta$ explicitly. An appropiate initial condition is obtained as follows:

Lemma 5.2. In points $p \in T$, we have

$$
\theta(p):=\operatorname{det}\left(\partial_{h} \psi_{0}(h, \mu) \mid X_{\mu}(p)\right)^{-1},
$$

with $h=H(p)$. (We write $\partial_{h} \psi_{0}(h, \mu)$ as the first column in a matrix, and $X_{\mu}(p)$ as the second column.)

Proof. There is a strong relation between $H$ and $\psi$, since

$$
H\left(\psi_{0}(h, \mu), \mu\right)=h .
$$

Derive this equation with respect to $h$ :

$$
D_{(x, y)} H\left(\psi_{0}(h, \mu), \mu\right) \partial_{h} \psi_{0}(h, \mu)=1 .
$$

Let $p=\psi_{0}(h, \mu)$, then the above equation becomes

$$
D_{(x, y)} H(p) \partial_{h} \psi_{0}(h, \mu)=1,
$$


or differently written

$$
\operatorname{det}\left(\partial_{h} \psi_{0}(h, \mu),\left(-\frac{\partial H}{\partial y}(p), \frac{\partial H}{\partial x}(p)\right)\right)=1 .
$$

Since $\theta f=-\frac{\partial H}{\partial y}$ and $\theta g=\frac{\partial H}{\partial x}$, it gives the result.

The problem with this result is that the initial condition $\theta$ depends on $\mu$. We will see that this is a problem that we would like to avoid. First, notice that if $\theta_{0}=\left.\theta\right|_{\mu=0}$, then

$$
X_{0}\left(\theta_{0}\right)=-\theta_{0} \operatorname{div} X_{0}
$$

so

$$
\theta_{0}(p)=\theta_{0}(P(p, 0)) \exp \left(\int_{O(p, 0)} \operatorname{div} X_{0}\right),
$$

where $O(p, 0)$ is the orbit along $X_{0}$ from $p$ to the intersection point $P(p, 0)$ of the orbit with $T$. Assuming we can calculate this integral explicitly, then we can move on to $\theta_{1}:=\left.\frac{\partial \theta}{\partial \mu}\right|_{\mu=0}$. However, plugging this into the equation, we see that at some point we need to calculate $X_{1}\left(\theta_{0}\right)$, with $X_{1}=\left.\frac{\partial X}{\partial \mu}\right|_{\mu=0}$.

This means that we must be able to derive $\theta_{0} \circ P$ as well as an integral along orbits of $X_{0}$. It is, however, possible to avoid deriving $\theta_{0} \circ P$, if we choose the section $T$ in such a way that $\theta_{0}$ does not depend on $\mu$. Since the choice of the section will not affect implicit results (such as the calculation of the control curve), we have some freedom in the choice of the coordinate system on $T$.

Lemma 5.3. On $T$, there exists a coordinate system (and an associated integrating factor $\theta$ on the blow up locus) so that for all points $p \in T$ close enough to the intersection point $\gamma \cap T$, we have

$$
\theta(p)=1 .
$$

Proof. Let $h=\alpha(k, \mu)$ be a regular change of coordinates, so that

$$
\psi_{\alpha}(k, \mu):=\left(\psi_{0}(\alpha(k, \mu), \mu), \mu\right)
$$

is a new coordinate function for $T$. We will put conditions on $\alpha$ so that $\theta$ with respect to this new coordinate function has the required property. According to Lemma 5.2, we need to solve

$$
g(p) \frac{\partial}{\partial k}\left(\psi_{0 x}(\alpha(k, \mu), \mu)\right)-f(p) \frac{\partial}{\partial k}\left(\psi_{0 y}(\alpha(k, \mu), \mu)\right)=1,
$$

where $p=\psi_{\alpha}(k, \mu)$. Working out the above expression yields a differential equation for $\alpha$ :

$$
\frac{d \alpha}{d k}=\left.\frac{1}{g(p) \frac{\partial \psi_{0 x}}{\partial h}(\alpha, \mu)-f(p) \frac{\partial \psi_{0 y}}{\partial h}(\alpha, \mu)}\right|_{p=\left(\psi_{0}(\alpha, \mu), \mu\right)} .
$$

The local existence of solutions of differential equations implies the result.

In these coordinates, we can calculate $\theta$ more easily. Writing

$$
X_{\mu}=X_{0}+\mu X_{1}+\mu^{2} X_{2}+\cdots
$$

and

$$
\theta_{\mu}=\theta_{0}+\mu \theta_{1}+\mu^{2} \theta_{2}+\cdots,
$$


we can give recursive formulas for $\theta_{i}$ (always under the assumption that $\mu$ is 1 dimensional):

$$
\sum_{i, j} X_{i}\left(\theta_{j}\right) \mu^{i+j}=\sum_{i, j}\left(-\theta_{j} \operatorname{div} X_{i}\right) \mu^{i+j}
$$

At zero order,

$$
\theta_{0}=\exp \left(\int_{O(p, 0)} \operatorname{div} X_{0}\right)
$$

with $O(p, 0)$ the orbit from $(p, 0)$ to the intersection point at $T$. For higher orders, we have to solve differential equations at each point, but an integration along orbits of $X_{0}$ will always lead to solutions. This knowledge combined with the fact that the initial conditions are trivial leads to a recursion only depending on the jets of $X_{\mu}$ along $\mu=0$. Note that the integrands at higher order could contain $X_{i}\left(\theta_{j}\right)$, so a way of deriving $\theta_{j}$ needs to be available to be able to calculate the integrals. All these integrals only involve the unperturbed vector field, but still they can be quite complicated.

Using (24), one finds

$$
\theta_{n}=\theta_{0} \int_{O(p, 0)} \sum_{k=0}^{n-1}\left(\theta_{k} / \theta_{0}\right)\left(\operatorname{div} X_{n-k}+X_{n-k}\left(\log \theta_{0}\right)+X_{n-k}\left(\theta_{k} / \theta_{0}\right)\right) .
$$

5.3. Heteroclinic connections on the blow up locus. Let us apply the results of Section 5.2 to the setting of this paper. Take a section $T$ transverse to the flow of $\bar{X}$ on the blow up locus (in the family rescaling chart), and assume $T$ intersects the heteroclinic connection $\Gamma$ which connects the points at infinity $P_{-}$and $P_{+}$.

Write

$$
\theta(\bar{x}, \bar{y}):=\exp \left(\left.\int_{\mathcal{O}(\bar{x}, \bar{y})} \operatorname{div} \bar{X}\right|_{u=A=0}\right)
$$

where $\mathcal{O}(\bar{x}, \bar{y})$ denotes the orbit along the unperturbed vector field from $(\bar{x}, \bar{y})$ to the intersection point in $T$. We have chosen a coordinate system on $T$ so that $\theta=1$ for points on $T$ (see Lemma 5.3 ).

Let $\zeta_{-}$be the intersection of an invariant manifold $W_{-}$from Section 3.3 with $T$, and let $\zeta_{+}$be the intersection of $W_{+}$with $T$ for some choices for the manifolds $W_{-}$and $W_{+}$. In the chosen coordinate system, let $z_{-}(u, A, \lambda)$ be the graph representation of $\zeta_{-}$and let $z_{+}(u, A, \lambda)$ be the graph representation of $\zeta_{+}$.

Following the techniques of the previous section, we can calculate the $k$-jets $j_{k}\left(z_{+}-z_{-}\right)$, with respect to $\mu$, provided that we are able to calculate $X_{0}(H)$ for a first integral $H$. The link between $X_{0}(H)$ and $\zeta_{ \pm}$is stated in a limit version of formula (23):

Lemma 5.4. Consider the vector field $\left.\bar{X}\right|_{A=0}$ with the perturbation parameter $u$. Then, in (23),

$$
\lim _{p \rightarrow P_{-}} j_{k}^{u} H(p, u)=j_{k}^{u} z_{-}(0, \lambda), \quad \lim _{q \rightarrow P_{+}} j_{k}^{u} H(q, u)=j_{k}^{u} z_{+}(0, \lambda),
$$

where $j_{k}^{u}$ is the $k$-jet w.r.t. $u$. Hence, also the right-hand side of (23) converges:

$$
\lim _{p \rightarrow P_{-}, q \rightarrow P_{+}} \int_{\gamma(p, q)} j_{k}^{\mu} X_{0}(H)=\int_{\Gamma} j_{k}^{\mu} X_{0}(H),
$$

where $\Gamma$ is the heteroclinic connection from $P_{-}$to $P_{+}$. 
Proof. Let us focus on $z_{-}$, and get a clear idea of what we have to prove. Given a point $p$ on the blow up locus, then the line segment $\{(p, u) \mid u \geq 0\}$ can be saturated with respect to $\bar{X}$. We have to prove that as $p$ gets closer to the end point $P_{-}$, the intersection of this saturation with $T$ is $O\left(u^{k}\right)$-close to any choice of invariant manifold $W_{-}$, and this for any $k>0$. Close enough to $P_{-}$the straight line segment in $C^{k}$-normal form coordinates is expressed by a $C^{k}$-curve $(\bar{v}(u), z(u))$ with $(\bar{v}(0), z(0))$ on the center manifold of $P_{-}$(the connection on the blow up locus). Choose a $C$ with $0<\bar{v}(0)<C$ and saturate (in normal form coordinates) the $C^{k}$-curve until the section $T^{\prime}:\{\bar{v}=C\}$ is met.

Take an initial point $\left(u_{0}, \bar{v}_{0}, z_{0}\right)$, and let $(u, v, z)$ be the coordinates of the intersection of the orbit through $\left(u_{0}, \bar{v}_{0}, z_{0}\right)$ with $T^{\prime}$. In the light of these remarks, we have to prove that $z=O\left(u^{k}\right)$.

It is easy to show that there is a $\kappa>0$ so that the transition time $\tau$ to go from a sufficiently small neighbourhood of $(0, \bar{v}(0), 0)$ to $T^{\prime}$ has a lower bound given by

$$
\tau \geq \frac{\kappa}{\bar{v}_{0}}
$$

Since $z(t)=z_{0} \exp (-t)$, we find $|z| \leq\left|z_{0}\right| \exp \left(-\kappa / \bar{v}_{0}\right)$. Finally, we have a first integral $u^{m} \bar{v}$, hence $u_{0}^{m} \bar{v}_{0}=u^{m} C$. We conclude

$$
|z| \leq\left|z_{0}\right| \exp \left(-\tilde{\kappa} / u^{1 / m}\right)
$$

for some $\tilde{\kappa}>0$. This proves the $k$-flatness to $z=0$ in the section $T^{\prime}$.

Lemma 5.5. Consider the vector field $\left.\bar{X}\right|_{u=0}$ with the perturbation parameter $A$. Then, in (23),

$$
\lim _{p \rightarrow P_{-}} j_{k}^{A} H(p, A)=j_{k}^{A} z_{-}(0, \lambda), \quad \lim _{q \rightarrow P_{+}} j_{k}^{A} H(p, A)=j_{k}^{A} z_{+}(0, \lambda),
$$

where $j_{k}^{A}$ is the $k$-jet w.r.t. $A$.

Proof. The technique of proving this lemma is slightly different. Let us again focus on $z_{-}$. Look at line segments $\{(p, A) \mid A \sim 0\}$. Taking the union for all $p$ on the heteroclinic connection, we get a manifold (not necessarily invariant) $U$. As in the previous lemma, we choose a section $T^{\prime}$ close to $P_{-}$, and look in the phase directional rescaling chart. Consider now the normal form of Lemma 3.3, restricted to $u=0$ and look in the $(\bar{v}, z, A)$-space:

$$
\left\{\begin{aligned}
\dot{A} & =0, \\
\dot{\bar{v}} & =m \bar{v}^{2} h(0, \bar{v}, A, \lambda), \\
\dot{z} & =-z .
\end{aligned}\right.
$$

In these coordinates, the manifold $U$ is a graph $z=\phi(\bar{v}, A)$, and any choice of invariant manifold $W$ is $O\left(A^{k}\right)$-close to $z=0$ (in fact $\{z=0\}$ is the unique center manifold for this system - all invariant manifolds $W$ have a common intersection with the blow up locus). The normal form is integrable: if an initial condition $\left(\bar{v}_{0}, z_{0}, A_{0}\right)$ inside $U$ is taken, and we intersect with a section $T^{\prime}:\{\bar{v}=C\}$, then we get

$$
A=A_{0}, \bar{v}=C, z=\phi\left(\bar{v}_{0}, A\right) \exp \left(-\int_{\bar{v}_{0}}^{C} \frac{d s}{m s^{2} h(0, s, A, \lambda)}\right) .
$$

Letting $\bar{v}_{0}$ tend to zero, one can prove that the $k$-jet of the last expression tends to zero as well (it is in fact a consequence of case (b) of Proposition A.1, if we apply the transformation $s=-\tilde{s}$ ). We conclude that the saturation of line pieces are 
$O\left(A^{k}\right)$-close to any choice of invariant manifold $W$ if the line pieces tend to the line of singularities.

Remark that the preceding two lemma's use techniques from the previous section, with $\mu=u$ or $\mu=A$ one-dimensional. If we want to examine $j_{k}^{u, A}$, then the previous section needs to be formulated in a more dimensional context.

Now, let us apply the results in the previous section, first on $\left.\bar{X}\right|_{A=0}$, and on $\left.\bar{X}\right|_{u=0}$. An immediate consequence of the above lemmas, and (23) is:

$$
\left(j_{k}^{u} z_{-}(0, \lambda)-j_{k}^{u} z_{+}(0, \lambda)\right)=\left.j_{k}^{u} \int_{\Gamma} \theta\left(\left.\bar{f}\right|_{u=0} \bar{g}-\left.\bar{g}\right|_{u=0} \bar{f}\right)\right|_{A=0}
$$

and

$$
\left(j_{k}^{A} z_{-}(0, \lambda)-j_{k}^{A} z_{+}(0, \lambda)\right)=\left.j_{k}^{A} \int_{\Gamma} \theta\left(\left.\bar{f}\right|_{A=0} \bar{g}-\left.\bar{g}\right|_{A=0} \bar{f}\right)\right|_{u=0}
$$

where $\Gamma$ is the heteroclinic connection for $u=A=0$.

Define the separation function

$$
\Delta(u, A, \lambda):=z_{-}(u, A, \lambda)-z_{+}(u, A, \lambda) .
$$

Then, using the $k$-jet formulas from above, we can easily find expressions for the 1-jet:

\section{Proposition 5.6.}

$$
\Delta(u, 0, \lambda)=u \int_{\Gamma} F_{u}+O\left(u^{2}\right)
$$

with

$$
F_{u}:=\left.\theta\left(\bar{f} \frac{\partial \bar{g}}{\partial u}-\bar{g} \frac{\partial \bar{f}}{\partial u}\right)\right|_{u=A=0}
$$

and with $\theta$ as in (26).

Proof. Immediate from (27), if one calculates the 1-jet of $\theta\left(\left.\bar{f}\right|_{u=0} \bar{g}-\left.\bar{g}\right|_{u=0} \bar{f}\right)$ with respect to $u$.

\section{Proposition 5.7.}

$$
\Delta(0, A, \lambda)=A \int_{\Gamma} F_{A}+O\left(A^{2}\right)
$$

with

$$
F_{A}:=\left.\theta\left(\bar{f} \frac{\partial \bar{g}}{\partial A}-\bar{g} \frac{\partial \bar{f}}{\partial A}\right)\right|_{u=A=0}
$$

and with $\theta$ as in (26). The parameter $A$ has the required regular breaking property if and only if

$$
\rho(\lambda)=\int_{\Gamma} F_{A} \neq 0
$$

where $\rho(\lambda)$ has been introduced in (8).

Combining these two propositions yields

Corollary 5.8. The first order term in $\mathcal{A}(u, \lambda)$ is

$$
\frac{\partial \mathcal{A}}{\partial u}(0, \lambda)=-\frac{\int_{\Gamma} F_{u}}{\int_{\Gamma} F_{a}},
$$

where $F_{u}$ and $F_{a}$ are defined above. 
5.4. Higher order angles. Assuming that the first order angle is zero, it may be interesting to find the first order contact that is nonzero. Note that even though the first order angle is zero, the invariant manifolds can be tilted; the angle in the forward manifolds is then the same as the angle in the backward manifolds. The appearance of a tilt will have an effect on higher order terms, and that is the reason why the knowledge of $z_{ \pm}$up to order $u^{k-1}$ is necessary to calculate the angle of order $u^{k}$. The method consists in calculating inductively the terms of order $u^{k}$ of the invariant manifolds, stopping at the first order where the angle has a nonzero coefficient. The method is based on the expansion of (27) in terms of $u$. This involves calculating the integrating factor $\theta$ up to order $u^{k-1}$, or at least $\left.\theta\right|_{\Gamma}$. To that end, the recursive formula (25) can be used, however, as one might expect, the formulas become quite cumbersome.

\section{Proof of Theorem 4}

Under the conditions of Theorem 4, we can explicitly calculate the optimal weights for the blow up and check the conditions for Theorems 1 and 2 . Let us first rewrite the vector field in some kind of standard form:

Lemma 6.1. Under the conditions of Theorem 4, $X_{\varepsilon, a, \lambda}$ is locally $C^{\infty}$ conjugate to

$$
\tilde{X}_{\varepsilon, a, \lambda}:\left\{\begin{aligned}
\dot{x} & =-y+\frac{1}{2} x^{2}+x^{3} F_{1}(x, y, \varepsilon, a, \lambda)+x \varepsilon F_{2}(x, y, \varepsilon, a, \lambda) \\
\dot{y} & =\varepsilon G(x, y, \varepsilon, a, \lambda) .
\end{aligned}\right.
$$

Furthermore,

(i) $G(0,0,0,0, \lambda)=-g_{0} f_{x x} f_{y}$;

(ii) $\frac{\partial G}{\partial x}(0,0,0,0, \lambda)=-g_{x} f_{y}$;

(iii) $\frac{\partial G}{\partial a}(0,0,0,0, \lambda)=g_{x}\left|\begin{array}{cc}f_{y} & f_{a} \\ f_{x y} & f_{x a}\end{array}\right|-f_{x x}\left|\begin{array}{cc}f_{y} & f_{a} \\ g_{y} & g_{a}\end{array}\right|$.

Proof. Consider a transformation of the form

$$
x=\alpha(y, a, \lambda) \tilde{x}+\beta(y, a, \lambda) .
$$

Since $\dot{y}=O(\varepsilon)$, we have $\dot{x}=\alpha \dot{\tilde{x}}+O(\varepsilon)$, and hence

$$
\begin{aligned}
\dot{\tilde{x}}= & \frac{1}{\alpha} f(\alpha \tilde{x}+\beta, y, 0, a, \lambda)+O(\varepsilon) \\
= & \frac{1}{\alpha} f(\beta, y, 0, a, \lambda)+\frac{\partial f}{\partial x}(\beta, y, 0, a, \lambda) \tilde{x} \\
& +\alpha \frac{\partial^{2} f}{\partial x^{2}}(\beta, y, 0, a, \lambda) \frac{\tilde{x}^{2}}{2}+O\left(\tilde{x}^{3}\right)+O(\varepsilon) .
\end{aligned}
$$

Consider the mapping

$$
I:(y, a, \lambda, \alpha, \beta) \mapsto\left(\frac{\partial f}{\partial x}(\beta, y, 0, a, \lambda), \alpha \frac{\partial^{2} f}{\partial x^{2}}(\beta, y, 0, a, \lambda)\right) .
$$

Defining $\alpha_{0}=1 / f_{x x}$, then $I\left(0,0, \lambda, \alpha_{0}, 0\right)=(0,1)$. Furthermore,

$$
D_{\alpha, \beta}(I)\left(0,0, \lambda, \alpha_{0}, 0\right)=\left(\begin{array}{cc}
0 & f_{x x} \\
f_{x x} & *
\end{array}\right) \text {. }
$$


This linear operator is invertible, hence the implicit function theorem gives us the existence of $(\alpha, \beta)$ so that

$$
\dot{\tilde{x}}=F(\tilde{x}, y, \varepsilon, a, \lambda):=\frac{1}{\alpha(y, a, \lambda)} f(\beta(y, a, \lambda), y, 0, a, \lambda)+\frac{\tilde{x}^{2}}{2}+O\left(\tilde{x}^{3}\right)+O(\varepsilon) .
$$

Because we need it for the second part, we will give the asymptotics of $\alpha$ and $\beta$ w.r.t. $(y, a)$ :

$$
\alpha(y, a, \lambda)=\left(\frac{1}{f_{x x}}\right)+O(\|(y, a)\|)
$$

and

$$
\beta(y, a, \lambda)=\left(\frac{-f_{x y}}{f_{x x}}\right) y+\left(\frac{-f_{x a}}{f_{x x}}\right) a+O\left(\|(y, a)\|^{2}\right) .
$$

Define now

$$
\tilde{y}:=-F(0, y, \varepsilon, a, \lambda)=\frac{-1}{\alpha(y, a, \lambda)} f(\beta(y, a, \lambda), y, 0, a, \lambda)+O(\varepsilon) .
$$

We can use $\tilde{y}$ as new $y$ coordinate, locally near $(y, \varepsilon, a)=(0,0,0)$. In this form, we find

$$
\dot{\tilde{x}}=-\tilde{y}+\frac{1}{2} \tilde{x}^{2}+O\left(\tilde{x}^{3}\right)+\varepsilon O(x) .
$$

This finishes the first part of the lemma. The second part is more elaborate. Let $y=\phi(\tilde{y}, \varepsilon, a, \lambda)$ be the implicit solution of (29), then

$$
\dot{\tilde{y}}=-\left.\varepsilon \frac{\partial F}{\partial y}(0, y, \varepsilon, a, \lambda) g(x, y, \varepsilon, a, \lambda)\right|_{y=\phi(\tilde{y}, \varepsilon, a, \lambda)} .
$$

So,

$$
G(\tilde{x}, \tilde{y}, \varepsilon, a, \lambda)=-\frac{\partial F}{\partial y}(0, \phi, 0, a, \lambda) g(\alpha \tilde{x}+\beta, \phi, 0, a, \lambda)+O(\varepsilon) .
$$

We can calculate $\frac{\partial F}{\partial y}$ (remembering that $\left.\frac{\partial f}{\partial x}(\beta(y, a, \lambda), y, 0, a, \lambda) \equiv 0\right)$ :

$$
\begin{aligned}
\frac{\partial F}{\partial y}(0, y, 0, a, \lambda)= & \frac{-1}{\alpha(y, a, \lambda)^{2}} f(\beta(y, a, \lambda), y, 0, a) \frac{\partial \alpha}{\partial y}(y, a, \lambda) \\
& +\frac{1}{\alpha} \frac{\partial f}{\partial y}(\beta(y, a, \lambda), y, 0, a, \lambda) .
\end{aligned}
$$

So,

$$
\frac{\partial F}{\partial y}(0,0,0,0, \lambda)=f_{x x} f_{y}
$$

From this last property easily follow the expressions for both $G(0,0,0,0, \lambda)$ and $\frac{\partial G}{\partial x}(0,0,0,0, \lambda)$ as claimed in the lemma. Let us now focus on $\frac{\partial G}{\partial a}(0,0,0,0, \lambda)$ :

$$
\begin{aligned}
\frac{\partial G}{\partial a}(0,0,0,0, \lambda)=g_{0} C(\lambda) & \\
-f_{x x} f_{y} & \left(g_{x}\left(\frac{\partial \beta}{\partial a}(0,0, \lambda)+\frac{\partial \beta}{\partial y}(0,0, \lambda) \frac{\partial \phi}{\partial a}(0,0,0, \lambda)\right)\right. \\
& \left.+g_{y} \frac{\partial \phi}{\partial a}(0,0,0, \lambda)+g_{a}\right),
\end{aligned}
$$

where $C(\lambda)$ is some function of which the value is irrelevant in view of proving the lemma, since under the assumptions of Theorem $4, g_{0}=0$ ). If we find the value 
of $\frac{\partial \phi}{\partial a}$, we can put all pieces together. It can be readily checked from formula (29) that

We conclude:

$$
\frac{\partial \phi}{\partial a}(0,0,0, \lambda)=-\frac{\frac{\partial F}{\partial a}(0,0,0,0, \lambda)}{\frac{\partial F}{\partial y}(0,0,0,0, \lambda)}=-\frac{f_{a}}{f_{y}}
$$

$$
\frac{\partial G}{\partial a}(0,0,0,0, \lambda)=g_{0} C(\lambda)-f_{x x} f_{y}\left(g_{x}\left(-\frac{f_{x a}}{f_{x x}}+\frac{f_{x y}}{f_{x x}} \frac{f_{a}}{f_{y}}\right)-g_{y} \frac{f_{a}}{f_{y}}+g_{a}\right) .
$$

Elaborating this expression yields a proof of the lemma.

Corollary 6.2. Under the assumptions of Theorem 4 , the family of vector fields $X_{\varepsilon, a, \lambda}$ is locally $C^{\infty}$-equivalent to

$$
\tilde{X}_{\varepsilon, a, \lambda}:\left\{\begin{aligned}
\dot{x} & =-y+\frac{1}{2} x^{2}+x^{3} F_{1, \lambda}(x, y, \varepsilon, a)+x \varepsilon F_{2, \lambda}(x, y, \varepsilon, a) \\
\dot{y} & =\varepsilon\left(a+x+G_{\lambda}(x, y, \varepsilon, a)\right)
\end{aligned}\right.
$$

with $F_{1, \lambda}, F_{2, \lambda}$ and $G_{\lambda} C^{\infty}$ functions and

$$
G(x, y, \varepsilon, a, \lambda)=O\left(\varepsilon, y,\|(x, a)\|^{2}\right),
$$

and where the $O$ notation is uniform in $\lambda$.

Proof. Take $G$ as in Lemma 6.1. From the assumptions of Theorem 4 we know that $\frac{\partial G}{\partial x}(0,0,0,0, \lambda)>0$. By rescaling $\varepsilon$ with a positive factor, we may assume that $\frac{\partial G}{\partial x}=-1$. We also know that $\frac{\partial G}{\partial a}(0,0,0,0, \lambda) \neq 0$. By rescaling the $a$-space with a nonzero factor, we may assume that this derivative is 1 . This proves the corollary.

Let us now check Assumptions 1-6 for the normal form (30). The first assumption - the existence of a critical curve - is guaranteed by the implicit function theorem:

$$
y-\frac{1}{2} x^{2}+x^{3} F_{1, \lambda}(x, y, 0,0)=0 .
$$

Clearly, there is a unique solution in the neighbourhood of $(x, y)=(0,0)$. Here, the curve is a graph $y=\phi(x)=\frac{1}{2} x^{2}+O\left(x^{3}\right)$. Looking at the linear part of (30), we find, for $\varepsilon=0$,

$$
\left(\begin{array}{cc}
x+O\left(x^{2}\right) & -1+O\left(x^{3}\right) \\
0 & 0
\end{array}\right) .
$$

The eigenspace transverse to the curve of singularities $\gamma$ has eigenvalue $x$, so for $x<0$ we have attraction, and for $x>0$ there is repulsion.

Assumption 2 can be checked as follows: Substituting $y=\phi(x)$ in $\frac{1}{\varepsilon} \dot{y}$ leads to the slow dynamics

$$
\phi^{\prime}(x) x^{\prime}=x+G_{\lambda}(x, \phi(x), 0,0) .
$$

Since $\phi^{\prime}(x)=x+O\left(x^{2}\right)$, we find

$$
x^{\prime}=1+O(x) .
$$

The slow dynamics ensures movement from the attracting to the repelling part of the critical curve.

To look at Assumption 3, we need to blow up the family of vector fields. We use a rescaling in the parameter space:

$$
a=v A, \quad \varepsilon=v^{2} .
$$


The parameter $A$ will serve as regular breaking parameter, but we come to that later. In terms of these new parameters, the vector field yields

$$
\left\{\begin{array}{l}
\dot{x}=-y+\frac{1}{2} x^{2}+x^{3} \tilde{F}_{1, \lambda}(x, y, v, A)+x v^{2} \tilde{F}_{2, \lambda}(x, y, v, A) \\
\dot{y}=v^{2}\left(v A+x+\tilde{G}_{\lambda}(x, y, v, A) .\right.
\end{array}\right.
$$

The blow up formulas for blowing up the origin are

$$
x=u \bar{x}, y=u^{2} \bar{y}, v=u \bar{v} .
$$

In the phase directional rescaling chart, we look at the directional chart $\bar{y}=1$, and find (after dividing through $u$ ) a family

$$
\left\{\begin{aligned}
\dot{u} & =\frac{1}{2} u \bar{v}^{2}(\bar{v} A+\bar{x}+O(u)), \\
\dot{\bar{v}} & =-\frac{1}{2} \bar{v}^{3}(\bar{v} A+\bar{x}+O(u)), \\
\dot{\bar{x}} & =-1+\frac{1}{2} \bar{x}^{2}-\frac{1}{2} \overline{x v}^{2}(\bar{v} A+\bar{x})+O(u) .
\end{aligned}\right.
$$

The preimage of the attracting part $\gamma_{-}$is represented by $\{\bar{x}=-\sqrt{2}+O(u), \bar{v}=0\}$, which is normally hyperbolically attracting with eigenvalue $\bar{x}<0$. Similarly, the preimage of the repelling part will be normally hyperbolic up to the end point $P_{+}$.

To check the connection assumption (Assumption 5), we look in the family rescaling chart $\bar{v}=1$. Here, the family of vector fields is equivalent to

$$
\left\{\begin{array}{l}
\dot{\bar{x}}=-\bar{y}+\frac{1}{2} \bar{x}^{2}+O(u) \\
\dot{\bar{y}}=A+\bar{x}+O(u)
\end{array}\right.
$$

The invariant line $\bar{y}=\frac{1}{2} \bar{x}^{2}-1$ is a curve without singularities, connecting $P_{-}$to $P_{+}$. To verify that $P_{ \pm}$is indeed a part of this line, one needs to look at $\bar{y}=\frac{1}{2} \bar{x}^{2}-1$ in the phase directional rescaling coordinates; there, this curve is represented by $\left\{\bar{x}^{2}=2\left(\bar{v}^{2}+1\right), u=0\right\}$. In any case, Assumption 5 is verified.

Assumption 6 can be checked easily in this case. This is because the unperturbed vector field is Hamiltonian in the family rescaling chart, with integrating factor

$$
\theta(\bar{x}, \bar{y})=\exp (-\bar{y})
$$

Using Proposition 5.7, the parameter $A$ is a regular breaking parameter if

$$
\int_{\Gamma}\left(-\bar{y}+\frac{1}{2} \bar{x}^{2}\right) \theta(\bar{x}, \bar{y}) \neq 0,
$$

where $\Gamma$ is the heteroclinic connection $\bar{y}=\frac{1}{2} \bar{x}^{2}-1$. The integral can be explicitly evaluated.

This proves the first part of Theorem 4 The next lemma deals with the second part of the proof of Theorem 4:

Lemma 6.3. There exist formal power series

$$
\hat{a}=\sum_{n=0}^{\infty} a_{n}(\lambda) \varepsilon^{n}, \quad \hat{y}=\sum_{n=0}^{\infty} y_{n}(x, \lambda) \varepsilon^{n}
$$

so that $y_{n}$ is smooth in a uniform neighbourhood of $x=0$, and so that $\hat{y}$ is formally invariant under (30).

Proof. For the sake of readibility, we drop the dependence on $\lambda$ in the notation. Let us first start by making an observation. Let $w(x, z, \varepsilon, a)$ be a smooth function 
in the neighbourhood of the origin. Then we can define

$$
\hat{w}(x, z, \varepsilon, a):=\sum_{|k|=0}^{\infty} w_{k}(x) z^{k_{1}} \varepsilon^{k_{2}} a^{k_{3}}
$$

where $k=\left(k_{1}, k_{2}, k_{3}\right)$ is a multi-index and the functions $w_{k}$ are defined in a uniform neighbourhood $\Omega$ of $x=0$. If we have formal power series $z=\hat{z}$ (w.r.t. $\varepsilon$ ) where the coefficients are also defined in $\Omega$, and if we have a formal power series $a=\hat{a}$, then we assert that $\hat{w}(x, \hat{z}, \varepsilon, \hat{a})$ makes sense, and is a formal power series where the coefficients are defined in the same neighbourhood $\Omega$, i.e. the neighbourhood does not shrink. This is true, provided that $\hat{z}$ and $\hat{a}$ have no terms in $\varepsilon^{0}$.

Keeping this property in mind, we write $y=\phi_{0}(x, a)+z$, where $y=\phi_{0}(x, a)$ is the graph of the curve of singularities of the unperturbed vector field $(\varepsilon=0)$ (the existence of $\phi_{0}$ follows from the implicit function theorem). In the search of a formal expansion of $z$, its constant term will be 0 . The formula for $\dot{x}$ yields

$$
\dot{x}=-z+x O(\varepsilon) \text {. }
$$

As for $\dot{z}$, we get

$$
\begin{aligned}
\dot{z} & =\dot{y}-\phi_{0}^{\prime}(x)(z+x O(\varepsilon)) \\
& =\varepsilon\left(a+x+O\left(\varepsilon, z,\|(x, a)\|^{2}\right)\right)-\phi_{0}^{\prime}(x)(-z+x O(\varepsilon)) .
\end{aligned}
$$

Knowing that $\phi_{0}^{\prime}(x)=x+O\left(x^{2}\right)$, we find

$$
\left\{\begin{array}{l}
\dot{x}=-z+x O(\varepsilon, a), \\
\dot{z}=\phi_{0}^{\prime}(x) z+\varepsilon\left(a+x+O\left(\varepsilon, z,\|(x, a)\|^{2}\right)\right) .
\end{array}\right.
$$

Solutions satisfy the differential equation:

$$
(-z+x O(\varepsilon, a)) \frac{d z}{d x}=\phi_{0}^{\prime}(x) z+\varepsilon\left(a+x+O\left(\varepsilon, z,\|(x, a)\|^{2}\right)\right) .
$$

Assume that $\hat{z}=\sum_{n=1}^{\infty} z_{n}(x) \varepsilon^{n}$ is known up to order $\varepsilon^{n}$ and $\hat{a}$ is known up to order $\varepsilon^{n-1}$. Look now at the coefficient of order $\varepsilon^{n+1}$ of the above equation. Remembering that $\hat{z}=O(\varepsilon)$ and $\hat{a}=O(\varepsilon)$, observe that the coefficient of order $\varepsilon^{n+1}$ of terms like $\hat{z}^{2}, \hat{z}^{3}, \varepsilon \hat{z}, \varepsilon^{2} \hat{a}$, etc. are functions in $x, z_{1}, \ldots, z_{n}, a_{1}, \ldots, a_{n-1}$. Hence, the term in $\varepsilon^{n+1}$ in the left-hand side of (32) is a function

$$
F_{n}\left(x, z_{1}, \ldots, z_{n}, \frac{d z_{1}}{d x}, \ldots, \frac{d z_{n}}{d x}, a_{1}, \ldots, a_{n-1}\right)+x H_{n}\left(x, a_{n}\right) \frac{d z_{1}}{d x} .
$$

Similarly, the right-hand side of (32) is of the form

$$
\phi_{0}^{\prime}(x) z_{n+1}+a_{n}+G_{n}\left(x, z_{1}, \ldots, z_{n}, \frac{d z_{1}}{d x}, \ldots, \frac{d z_{n}}{d x}, a_{1}, \ldots, a_{n-1}\right)
$$

for some function $G_{n}$. Assuming that $z_{1}, \ldots, z_{n}$ and $a_{1}, \ldots, a_{n-1}$ are already known, looking at the $\varepsilon^{n+1}$ level in (32) yields an equation

$$
\tilde{F}_{n}(x)+x H_{n}\left(x, a_{n}\right) \frac{d z_{1}}{d x}=\phi_{0}^{\prime}(x) z_{n+1}+a_{n}+\tilde{G}_{n}(x) .
$$

From the above equation, one can find a unique $a_{n}$, by reducing the equation to $x=0$, and once $a_{n}$ is known, the equation becomes

$$
\phi_{0}^{\prime}(x) z_{n+1}=O(x)
$$


From this equation, a smooth $z_{n+1}$ can be found, since $\phi_{0}^{\prime}(x)=x+O\left(x^{2}\right)$. This process is a recursion to find unique $\hat{a}$ and $\hat{z}$ as formal power series in $\varepsilon$. The final step will define $\hat{y}$ :

$$
\hat{y}=\phi(x, \hat{a})+\hat{z}
$$

which still will be a formal power series, with coefficients smooth in a uniform neighbourhood of $x=0$.

\section{Proof of Theorem 5}

Under the conditions of Theorem 5, we can explicitly calculate the optimal weights for the blow up and check the conditions for Theorem 11. Let us first rewrite the vector field in some normal form:

Lemma 7.1. Under the conditions of Theorem 5, $X_{\varepsilon, a, \lambda}$ is locally $C^{\infty}$ conjugate to

$$
\tilde{X}_{\varepsilon, a, \lambda}:\left\{\begin{aligned}
\dot{x} & =-y+\frac{1}{2 n} x^{2 n}+x F(x, y, \varepsilon, a, \lambda) \\
\dot{y} & =\varepsilon G(x, y, \varepsilon, a, \lambda)
\end{aligned}\right.
$$

with $F(x, 0,0,0, \lambda)=O\left(x^{2 n}\right)$. Furthermore,

(i) $F(0,0,0, a, \lambda)=\left(\frac{1}{f_{y}}\left|\begin{array}{cc}f_{y} & f_{a} \\ f_{x y} & f_{x a}\end{array}\right|\right) a+O\left(a^{2}\right)$;

(ii) $G(x, 0,0,0, \lambda)=\left(\frac{-1}{\alpha^{2 n-2}} f_{y} \frac{g_{x^{2 n-1}}}{(2 n-1) !}\right) x^{2 n-1}+O\left(x^{2 n}\right)$;

(iii) $G(0,0,0, a, \lambda)=\left(-\alpha\left|\begin{array}{ll}f_{y} & f_{a} \\ g_{y} & g_{a}\end{array}\right|\right) a+O\left(a^{2}\right)$,

with $\alpha=\left(\frac{f_{x 2 n}}{(2 n-1) !}\right)^{1 /(2 n-1)}$.

Proof. The proof is straightforward. Apply the transformation

$$
\tilde{x}=\alpha x, \quad \tilde{y}=-\alpha f(0, y, \varepsilon, a, \lambda)
$$

for some well-chosen number $\alpha$. If $\alpha$ is nonzero, then we can use $(\tilde{x}, \tilde{y})$ locally as new variables, since we know that $f_{y} \neq 0$. Let us calculate the new vector field:

$$
\dot{\tilde{x}}=\alpha f\left(\frac{1}{\alpha} \tilde{x}, y, \varepsilon, a, \lambda\right)=\alpha f(0, y, \varepsilon, a, \lambda)+O(\tilde{x})=-\tilde{y}+\tilde{F}(\tilde{x}, \tilde{y}, \varepsilon, a)
$$

for some function $\tilde{F}=O(\tilde{x})$. The terms of $O(\tilde{x})$ can be specified a bit more: from the conditions of Theorem 5 we get (using the notation introduced in the announcement of this theorem)

$$
\tilde{F}(\tilde{x}, 0,0,0, \lambda)=\frac{1}{\alpha^{2 n-1}} f_{x^{2 n}} \frac{\tilde{x}^{2 n}}{(2 n) !}+O\left(\tilde{x}^{2 n+1}\right) .
$$

Choose $\alpha=\left(\frac{f_{x^{2 n}}}{(2 n-1) !}\right)^{1 /(2 n-1)}$ so that $\tilde{F}(\tilde{x}, 0,0,0, \lambda)=\frac{\tilde{x}^{2 n}}{2 n}+O\left(\tilde{x}^{2 n+1}\right)$, and conclude:

$$
\dot{\tilde{x}}=-\tilde{y}+\frac{x^{2 n}}{2 n}+\tilde{x} F(\tilde{x}, \tilde{y}, \varepsilon, a, \lambda)
$$

with $F(\tilde{x}, 0,0,0, \lambda)=O\left(x^{2 n}\right)$. This finishes the first part of the lemma. The second part is more elborate. Let $y=\phi(\tilde{y}, \varepsilon, a, \lambda)$ be the implicit solution of 
$\tilde{y}=\alpha f(0, y, \varepsilon, a, \lambda)$, then

$$
\begin{aligned}
\frac{\partial F}{\partial a}(0,0,0,0, \lambda) & =\frac{\partial^{2} \tilde{F}}{\partial \tilde{x} \partial a}(0,0,0,0, \lambda) \\
& =\frac{\partial^{2} f}{\partial x \partial a}(0,0,0,0, \lambda)+\frac{\partial^{2} f}{\partial x \partial y}(0,0,0,0, \lambda) \frac{\partial \phi}{\partial a}(0,0,0, \lambda) \\
& =f_{x a}+f_{x y} \frac{\partial \phi}{\partial a}(0,0,0, \lambda) .
\end{aligned}
$$

From $\tilde{y}=-\alpha f(0, \phi(\tilde{y}, \varepsilon, a, \lambda), \varepsilon, a, \lambda)$, we obtain $f_{a}+f_{y} \frac{\partial \phi}{\partial a}(0,0,0, \lambda)=0$, and hence

$$
\frac{\partial F}{\partial a}(0,0,0,0, \lambda)=\frac{1}{f_{y}}\left(f_{x a} f_{y}-f_{x y} f_{a}\right) .
$$

Let us now concentrate on $\dot{\tilde{y}}$ : $\dot{\tilde{y}}=\varepsilon G(\tilde{x}, \tilde{y}, \varepsilon, a, \lambda)$, with

$$
G(\tilde{x}, \tilde{y}, \varepsilon, a, \lambda)=-\alpha \frac{\partial f}{\partial y}(0, \phi(\tilde{y}, \varepsilon, a, \lambda), \varepsilon, a, \lambda) g\left(\frac{1}{\alpha} \tilde{x}, \phi(\tilde{y}, \varepsilon, a, \lambda), \varepsilon, a, \lambda\right) .
$$

Hence,

$$
G(\tilde{x}, 0,0,0, \lambda)=-\alpha f_{y} g\left(\frac{1}{\alpha} \tilde{x}, 0,0,0, \lambda\right)=\frac{-1}{\alpha^{2 n-2}} f_{y} g_{x^{2 n-1}} \frac{x^{2 n-1}}{(2 n-1) !}+O\left(x^{2 n}\right) .
$$

Finally, it is easy to calculate that

$$
\frac{\partial G}{\partial a}(0,0,0,0, \lambda)=-\alpha f_{y}\left(g_{y} \frac{-f_{a}}{f_{y}}+g_{a}\right)=\alpha\left(g_{y} f_{a}-g_{a} f_{y}\right) .
$$

This finishes the proof.

Corollary 7.2. Under the assumptions of Theorem [5, the family of vector fields $X_{\varepsilon, a, \lambda}$ is locally $C^{\infty}$-equivalent to

$$
\tilde{X}_{\varepsilon, a, \lambda}:\left\{\begin{array}{l}
\dot{x}=-y+\frac{x^{2 n}}{2 n}+x F_{1}(x, y, \varepsilon, a, \lambda), \\
\dot{y}=\varepsilon\left(C a+x^{2 n-1}+G_{1}(x, y, \varepsilon, a, \lambda)\right),
\end{array}\right.
$$

with $F_{1}(x, 0,0,0, \lambda)=O\left(x^{2 n}\right)$, and $G_{1}(x, y, \varepsilon, a, \lambda)=O\left(x^{2 n}, a^{2}, y, \varepsilon\right)$. The constant $C$ may depend on $\lambda$, and the regular breaking condition in Theorem 5 is translated to

$$
C \neq \frac{\partial F_{1}}{\partial a}(0,0,0,0, \lambda)
$$

Proof. Take $F$ and $G$ as in Lemma 7.1. From the assumptions of Theorem 15, we know that the coefficient $\left(\frac{-1}{\alpha^{2 n-2}} f_{y} \frac{g_{x 2 n-1}}{(2 n-1) !}\right)$ is strictly positive; hence by rescaling $\varepsilon$ with this the positive factor $\left(\frac{-1}{\alpha^{2 n-2}} f_{y} \frac{g_{x 2 n-1}}{(2 n-1) !}\right)$, we reduce the coefficient of $x^{2 n-1}$ in $G$ to +1 . The coefficient of $a$ in $G$ will change to

$$
\frac{\left(-\alpha\left|\begin{array}{ll}
f_{y} & f_{a} \\
g_{y} & g_{a}
\end{array}\right|\right)}{-\left(\frac{-1}{\alpha^{2 n-2}} f_{y} \frac{g_{x^{2 n-1}}}{(2 n-1) !}\right)}=\frac{\alpha^{2 n-1}(2 n-1) !}{f_{y} g_{x^{2 n-1}}}\left|\begin{array}{l}
f_{y} f_{a} \\
g_{y} \\
g_{a}
\end{array}\right| .
$$

Since $\alpha^{2 n-1}=f_{x^{2 n}} /(2 n-1)$ !, the coefficient of $a$ in $G$ equals

$$
C:=\frac{f_{x^{2 n}}}{f_{y} g_{x^{2 n-1}}}\left|\begin{array}{cc}
f_{y} & f_{a} \\
g_{y} & g_{a}
\end{array}\right| .
$$

The regular breaking condition in Theorem 5 states that this coefficient $C$ cannot be equal to $\frac{1}{f_{y}}\left|\begin{array}{cc}f_{y} & f_{a} \\ f_{x y} & f_{x a}\end{array}\right|$ which is exactly the coefficient in $a$ of $F_{1}$. 
Let us now check Assumptions 1-6 for the normal form (33). The first assumption - the existence of a critical curve - is guaranteed by the implicit function theorem: we search $y$ in terms of $x$ so that

$$
y-\frac{1}{2 n} x^{2 n}+x F_{1}(x, y, 0,0, \lambda)=0 .
$$

Clearly, there is a unique graph solution in the neighbourhood of $(x, y)=(0,0)$. Here, the curve is a graph $y=\phi(x)=\frac{1}{2 n} x^{2 n}+O\left(x^{2 n+1}\right.$ ) (to see this, remember that $\left.F_{1}(x, 0,0,0, \lambda)=O\left(x^{2 n}\right)\right)$. Looking at the linear part of (33), we find, for $\varepsilon=0$

$$
\left(\begin{array}{cc}
x^{2 n-1}+O\left(x^{2 n}\right) & -1+O(x) \\
0 & 0
\end{array}\right) .
$$

The eigenspace transverse to the curve of singularities $\gamma$ has a negative eigenvalue for $x<0$, so there we have attraction, and for $x>0$ there is repulsion.

To look at Assumption 3, we need to blow up the family of vector fields. We use a rescaling in the parameter space

$$
a=v^{2 n-1} A, \quad \varepsilon=v^{2 n} .
$$

The parameter $A$ will serve as regular breaking parameter, but we come to that later. We then blow the origin, as follows:

$$
x=u \bar{x}, y=u^{2 n} \bar{y}, v=u \bar{v} .
$$

In the phase directional rescaling chart, we look at the directional chart $\bar{y}=1$, and find (after dividing through $u^{2 n-1}$ ) a family

$$
\left\{\begin{aligned}
\dot{u} & =\frac{1}{2 n} u \bar{v}^{2 n}\left(C \bar{v}^{2 n-1} A+\bar{x}^{2 n-1}+O(u)\right) \\
\dot{\bar{v}} & =-\frac{1}{2 n} \bar{v}^{2 n+1}\left(C \bar{v}^{2 n-1} A+\bar{x}^{2 n-1}+O(u)\right) \\
\dot{\bar{x}} & =-1+\frac{1}{2 n} \bar{x}^{2 n}+D A \bar{x}-\frac{1}{2 n} \overline{x v}^{2 n}\left(C \bar{v}^{2 n-1} A+\bar{x}^{2 n-1}\right)+O(u),
\end{aligned}\right.
$$

with

$$
D=\frac{\partial F_{1}}{\partial a}(0,0,0,0, \lambda)
$$

The preimage of the attracting part $\gamma_{-}$is represented by $\{\bar{x}=-\sqrt[2 n]{2 n}+O(u), \bar{v}=$ $0\}$, which is normally hyperbolically attracting, with eigenvalue $-\bar{x}^{2 n-1}$. Similarly, the preimage of the repelling part will be normally hyperbolicly repelling up to the end point $P_{+}$.

To check the connection assumption (Assumption 5), we look in the family rescaling chart $\bar{v}=1$. Here, the family of vector fields is equivalent to

$$
\left\{\begin{array}{l}
\dot{\bar{x}}=-\bar{y}+\frac{1}{2 n} \bar{x}^{2 n}+D \bar{x} A+O(u) \\
\dot{\bar{y}}=C A+\bar{x}^{2 n-1}+O(u) .
\end{array}\right.
$$

The invariant line $\bar{y}=\frac{1}{2 n} \bar{x}^{2 n}-1$ is a curve without singularities, connecting $P_{-}$to $P_{+}$. To verify that $P_{ \pm}$is indeed a part of this line, one needs to look at $\bar{y}=\frac{1}{2 n} \bar{x}^{2 n}-1$ in the phase directional rescaling coordinates; there, this curve is represented by $\left\{\bar{x}^{2 n}=2 n\left(\bar{v}^{2 n}+1\right), u=0\right\}$. In any case, Assumption 5 is verified.

Assumption 6 can be easily checked in this case. This is because the unperturbed vector field is Hamiltonian in the family rescaling chart, with integrating factor

$$
\theta(\bar{x}, \bar{y})=\exp (-\bar{y})
$$


Using Proposition 5.7 the parameter $A$ is a regular breaking parameter if

$$
\int_{\Gamma}\left(C\left(-\bar{y}+\frac{1}{2 n} \bar{x}^{2 n}\right)-D \bar{x}^{2 n}\right) \theta(\bar{x}, \bar{y}) \neq 0,
$$

where $\Gamma$ is the heteroclinic connection $\bar{y}=\frac{1}{2 n} \bar{x}^{2 n}-1$. The integral can be explicitly evaluated:

$$
\int_{\Gamma}\left(C\left(-\bar{y}+\frac{1}{2 n} \bar{x}^{2 n}\right)-D \bar{x}^{2 n}\right) \theta(\bar{x}, \bar{y})=(C-D) e \int_{-\infty}^{\infty} \exp \left(-s^{2 n} / 2 n\right) d s,
$$

where $e$ is the Euler number. Hence, the regular breaking condition is satisfied provided $C \neq D$.

This proves Theorem 5 .

\section{EXAmples}

The examples in this section are not meant to describe general classes of vector fields, but are aimed at illustrating in a rather unexpected way the theorems.

8.1. $C^{1}$ canard solutions. Consider

$$
X_{\varepsilon, a}:\left\{\begin{aligned}
\dot{x} & =y-\frac{x^{4}}{4}+\varepsilon x^{2}, \\
\dot{y} & =\varepsilon\left(a-x^{3}\right) .
\end{aligned}\right.
$$

According to Theorem 5 and Theorem 1 there exist manifolds of canard solutions that are $C^{\infty}$ in the blow up space. Also the control curves related to $C^{\infty}$ boundary conditions $\Sigma_{-}$and $\Sigma_{+}$are $C^{\infty}$ in $\varepsilon^{1 / 4}$. We show here that these manifolds can be blown down in a $C^{1}$ way, but not in a $C^{2}$ way, although the control curve itself will be smooth. Indeed, first notice that

$$
y=\frac{x^{4}}{4}-\left(1+x^{2}\right) \varepsilon+O\left(\varepsilon^{2}\right), a=O(\varepsilon)
$$

is a graph that is formally invariant w.r.t. $X_{\varepsilon, a}$, up to order $O(\varepsilon)$. Let us try to extend this to an expansion

$$
y=\frac{x^{4}}{4}-\left(1+x^{2}\right) \varepsilon+y_{2}(x) \varepsilon^{2}+O\left(\varepsilon^{3}\right), \quad a=a_{1} \varepsilon+O\left(\varepsilon^{2}\right) .
$$

Expressing the formal invariance of this new expansion quickly yields

$$
y_{2}(x)=\frac{a_{1}-2 x}{x^{3}} .
$$

Hence, no choice of $\left(a_{1}, y_{2}\right)$ exists so that the formal invariance is true up to order $O\left(\varepsilon^{2}\right)$ and so that $y_{2}$ is continuous at the origin.

But there is one more interesting observation to be made about this family of vector fields: for $a=0$, the vector field $X_{\varepsilon, a}$ has a symmetry $\{x \mapsto-x, t \mapsto$ $-t\}$. This means that if the two curves $\Sigma_{-}$and $\Sigma_{+}$in Theorem 1 are chosen symmetrically with respect to the $y$-axis, then the control curve will be situated at $\{a=0\}$ ! As a consequence, all control curves will be flat to $a=0$. Combine this with the knowledge that the control curves are smooth in $\varepsilon^{1 / 4}$, and we can conclude that each choice of control curve $a=\mathcal{A}(\varepsilon)$ will be $C^{\infty}$ in $\varepsilon$ !

Also, if the two boundary curves $\Sigma_{-}$and $\Sigma_{+}$are not chosen "symmetrically" $\left(\Sigma_{-}\right.$ is not inside the backward saturation of $\Sigma_{+}$with respect to $X_{\varepsilon, 0}$, or equivalently $\Sigma_{+}$is not inside the forward saturation of $\Sigma_{-}$w.r.t. $X_{\varepsilon, 0}$ ), then the control curve cannot be analytic, even if we know that the vector field and the boundary curves are analytic. 
8.2. Normal crossing of lines of singularities. Consider the scalar o.d.e.

$$
\varepsilon \frac{d y}{d x}=a+x^{2 n-1} y+\varepsilon F(x, y, \varepsilon, a, \lambda)
$$

and the associated vector field

$$
X_{\varepsilon, a, \lambda}:\left\{\begin{aligned}
\dot{x} & =\varepsilon \\
\dot{y} & =a+x^{2 n-1} y+\varepsilon F(x, y, \varepsilon, a, \lambda),
\end{aligned}\right.
$$

with $F$ a $C^{\infty}$ function in the neighbourhood of $(x, y, \varepsilon, a)=(0,0,0,0)$. For $\varepsilon=a=$ 0 , we have a crossing of the lines of singularities: $x=0$ and $y=0$. Along $x=0$, it would not be possible to satisfy the regular passage property, but along $y=0$, it will be, so we will define $y=0$ as the critical curve.

Observe that along $y=0$, for $x<0$ the critical curve is attracting, and for $x>0$ the critical curve is repelling. Given a point $p$ on the attracting part of the critical curve, then the regular passage assumption (Assumption 2) is trivially satisfied, since $\dot{x}=\varepsilon>0$. To check the remaining assumptions, we blow up the origin:

$$
\varepsilon=v^{4 n}, a=v^{4 n-1} A, \quad x=u^{2} \bar{x}, y=u \bar{y}, v=u \bar{v} .
$$

In the phase-directional rescaling chart $\bar{x}=+1$, we find (after dividing by $u^{4 n-2}$ )

$$
\left\{\begin{aligned}
\dot{u} & =\frac{1}{2} u \bar{\varepsilon}^{4 n} \\
\dot{\bar{v}} & =-\frac{1}{2} \bar{v}^{4 n+1}, \\
\dot{\bar{y}} & =\bar{v}^{4 n-1} A+\bar{y}-\frac{1}{2} \bar{v}^{4 n} \bar{y}+O(u) .
\end{aligned}\right.
$$

Clearly, Assumption 3 is satisfied, and due to the absence of singularities outside $u \bar{v}=0$ Assumption 4 also holds. Look now in the family rescaling chart $\bar{v}=1$ :

$$
\left\{\begin{array}{l}
\dot{\bar{x}}=1 \\
\dot{\bar{y}}=A+\bar{x}^{2 n-1} \bar{y}+O(u) .
\end{array}\right.
$$

Clearly, for $A=0$ there is a connection $\bar{y}=0$ connecting $P_{-}$to $P_{+}$, and on this connection, no singularities appear. This shows that Assumption 5 is satisfied. Finally, in order to check Assumption 6, one needs to calculate an integrating factor. One readily checks that

$$
\theta(\bar{x}, 0)=\exp \left(\int_{\bar{x}}^{0} s^{2 n-1} d s\right)=\exp \left(-\bar{x}^{2 n} / 2 n\right) .
$$

Using Proposition 5.7 $A$ is a regular breaking parameter because

$$
\int_{\bar{y}=0} \theta \neq 0
$$

The control curve $A=\mathcal{A}(u)$ in original coordinates is a curve

$$
a(\varepsilon)=\varepsilon^{(4 n-1) / 4 n} \mathcal{A}\left(\varepsilon^{1 / 4 n}\right) .
$$

where $\mathcal{A}$ is smooth in its argument. If $n>1$, then generally, $a(\varepsilon)$ will not be $C^{\infty}$ in $\varepsilon$. If $n=1$, then one can prove the existence of a formally invariant expansion, and using Theorem 2, the smoothness in $\varepsilon$ can be shown. 


\section{APPENDIX}

Proposition A.1. Let $g$ be a positive (not necessarily strictly positive) $C^{k}$-function on $V \times W \times \Lambda$, where $V$ is a compact interval in $\mathbb{R}, W$ is a set of singular parameters (part of a finitely dimensional vector space), and $\Lambda$ is a set of regular parameters (part of a finitely dimensional vector space). Define for a fixed $y_{0} \in V$, and for all $y>y_{0}$ :

$$
w(y, \varepsilon, \lambda):=\exp \left(-\int_{y_{0}}^{y} \frac{d s}{g(s, \varepsilon, \lambda)}\right), \quad \varepsilon \in W, \lambda \in \Lambda .
$$

Assume for $y_{1}>y_{0}$ that $\left(y_{1}, \varepsilon_{0}, \lambda\right)$ is in the closure of the domain of $w$. If

(a) $g\left(s, \varepsilon_{0}, \lambda\right)=0, \forall s \in\left[y_{0}, y_{1}\right]$

(b) or if $g\left(s, \varepsilon_{0}, \lambda\right)$ is only zero in the end point $s=y_{1}$ and not in $\left[y_{0}, y_{1}[\right.$, and if $\frac{\partial g}{\partial y}\left(y_{1}, \varepsilon_{0}, \lambda\right)=0$,

then the function $w$ can be extended in a $C^{k}$ way to $\left(y_{1}, \varepsilon_{0}, \lambda\right)$, and in this point $w$ and all its derivatives (up to order $k$ ) are zero.

Proof. We will first treat case (a), and then tell how to adapt the proof for case (b). We claim that it suffices to prove that

(P1) for all $N \in \mathbb{N}: \quad \lim _{(y, \varepsilon) \rightarrow\left(y_{1}, \varepsilon_{0}\right)} \frac{w(y, \varepsilon, \lambda)}{g(y, \varepsilon, \lambda)^{N}}=0$;

(P2) for all $K, M \in \mathbb{N}$ :

$$
\lim _{(y, \varepsilon) \rightarrow\left(y_{1}, \varepsilon_{0}\right)} w(y, \varepsilon, \lambda) g(y, \varepsilon, \lambda)^{K M}\left(\int_{y_{0}}^{y} \frac{d s}{g(s, \varepsilon, \lambda)^{K}}\right)^{M}=0,
$$

and where the convergence is uniform in $\lambda$. Note that the limits can only be taken in the closure of the set of points $(y, \varepsilon, \lambda)$ where $w(y, \varepsilon, \lambda)$ is defined properly. Note also that we do not claim anything for $y=y_{0}$; indeed this point is excluded in the formulation of the proposition. In fact, $w$ is in general not even $C^{1}$ in the point $\left(y_{0}, \varepsilon_{0}, \lambda\right)$.

The proof of properties (P1) and (P2) will be carried out in two lemma's, but here we will show that those two properties are sufficient to prove the proposition.

To that end, calculate all first-order derivatives of $w$, in the points $(y, \varepsilon, \lambda)$ where $w$ is defined:

$$
\begin{aligned}
& \frac{\partial w}{\partial y}(y, \varepsilon, \lambda)=w(y, \varepsilon, \lambda) \frac{-1}{g(y, \varepsilon, \lambda)}, \\
& \frac{\partial w}{\partial \varepsilon}(y, \varepsilon, \lambda)=w(y, \varepsilon, \lambda) \int_{y_{0}}^{y} \frac{\frac{\partial g}{\partial \varepsilon}(s, \varepsilon, \lambda)}{g(s, \varepsilon, \lambda)^{2}} d s, \\
& \frac{\partial w}{\partial \lambda_{\ell}}(y, \varepsilon, \lambda)=w(y, \varepsilon, \lambda) \int_{y_{0}}^{y} \frac{\frac{\partial g}{\partial \lambda_{\ell}}(s, \varepsilon, \lambda)}{g(s, \varepsilon, \lambda)^{2}} d s .
\end{aligned}
$$

Observing these three equations, one finds that applying a general differential operator $D$ to $w$ results in

$$
D w(y, \varepsilon, \lambda)=\sum_{i} w(y, \varepsilon, \lambda) \prod_{j} \frac{F_{i j}(y, \varepsilon, \lambda)}{g(y, \varepsilon, \lambda)^{N_{i j}}} \int_{y_{0}}^{y} \frac{G_{i j}(s, \varepsilon, \lambda)}{g(s, \varepsilon, \lambda)^{K_{i j}}} d s,
$$

with $N_{i j}, K_{i j} \in \mathbb{N}, F_{i j}$ and $G_{i j}$ are functions of class $C^{k-|D|}$ and where the sum and products are finite. In order to prove that $D w(y, \varepsilon, \lambda) \rightarrow 0$ as $\varepsilon \rightarrow \varepsilon_{0}$, it is sufficient to prove that each summand of the above expression tends to zero. By 
raising these expressions to some power, we can distribute the effect of $w(y, \varepsilon, \lambda)$ among all types of factors, and we find the following two conditions:

(a) for all $N \in \mathbb{N}$ : $\lim _{(y, \varepsilon) \rightarrow\left(y_{1}, \varepsilon_{0}\right)} \frac{w(y, \varepsilon, \lambda) F(y, \varepsilon, \lambda)}{g(y, \varepsilon, \lambda)^{N}}=0$;

(b) for all $K, M \in \mathbb{N}$ :

$$
\lim _{(y, \varepsilon) \rightarrow\left(y_{1}, \varepsilon_{0}\right)} w(y, \varepsilon, \lambda) g(y, \varepsilon, \lambda)^{K M}\left(\int_{y_{0}}^{y} \frac{G(s, \varepsilon, \lambda)}{g(s, \varepsilon, \lambda)^{K}} d s\right)^{M}=0
$$

(the factor $g^{K M}$ is included in (b) because we want to do so, and we can: we simply need to increase the $N$ in (a)). Since $F$ and $G$ are at least $C^{0}$, these properties are true once the two properties (P1) and (P2) are satisfied.

The remainder of the section involves the proof of (P1) and (P2). Let us start with

Lemma A.2 (Under condition (a) in Proposition A.1). For all $K>0$ there exists a neighbourhood $V$ of $\varepsilon=\varepsilon_{0}$ such that

$$
\int_{y_{0}}^{y} \frac{d s}{g(s, \varepsilon, \lambda)} \geq-K\left(y-y_{0}\right) \log g(y, \varepsilon, \lambda),
$$

for all $y \in\left[y_{0}, y_{1}\right], \lambda \in \Lambda$ and $\varepsilon \in V$ (only for those $\varepsilon$ where $w$ is defined).

Proof. Let

$$
F:(y, \varepsilon, \lambda) \mapsto \int_{y_{0}}^{y} \frac{d s}{g(s, \varepsilon, \lambda)}+K\left(y-y_{0}\right) \log g(y, \varepsilon, \lambda)
$$

Then $F\left(y_{0}, \varepsilon, \lambda\right)=0$, so it remains to prove that $\frac{\partial F}{\partial y}(y, \varepsilon, \lambda) \geq 0$ for all $y \in\left[y_{0}, y_{1}\right]$. In short notation, we have

$$
\begin{aligned}
\frac{\partial F}{\partial y} & =\frac{1}{g}+K \log g+\frac{K\left(y-y_{0}\right)}{g} \frac{\partial g}{\partial y} \\
& =\frac{1}{g}\left(1+K g \log g+K\left(y-y_{0}\right) \frac{\partial g}{\partial y}\right) .
\end{aligned}
$$

The mapping $u \mapsto u \log u$ tends to zero in the origin, so for $\varepsilon$ small enough, we may assume that $g \log g \geq-\frac{1}{3 K}$. Furthermore, since also $\frac{\partial g}{\partial y}$ tends to zero, we may assume that for $\varepsilon$ small enough, $\frac{\partial g}{\partial y} \geq-\frac{1}{3 K\left(y_{1}-y_{0}\right)}$. Applying these inequalities to the equation above, we find

$$
\frac{\partial F}{\partial y}(y, \varepsilon, \lambda) \geq \frac{1}{g(y, \varepsilon, \lambda)}\left(1+K \frac{-1}{3 K}+\left(y-y_{0}\right) K \frac{-1}{3 K\left(y_{1}-y_{0}\right)}\right) .
$$

As $y-y_{0} \leq y_{1}-y_{0}$, we have $\frac{\partial F}{\partial y}(y, \varepsilon, \lambda) \geq \frac{1}{3 g(y, \varepsilon, \lambda)} \geq 0$.

Corollary A.3. For all $N \in \mathbb{N}$ and for all intervals $\left[y_{0}+\delta, y_{1}\right]$ on the $y$-axis (with $\delta>0$ ), there exists a neighbourhood $V$ of $\varepsilon=\varepsilon_{0}$ such that

$$
w(y, \varepsilon, \lambda) \leq g(y, \varepsilon, \lambda)^{N+1},
$$

for all $y \in\left[y_{0}+\delta, y_{1}\right], \lambda \in \Lambda$ and $\varepsilon \in V$ (only for those $\varepsilon$ where $w$ is defined). This proves property $(\mathrm{P} 1)$ in case (a). 
Proof. Apply Lemma A.2 with $K=\frac{N+1}{\delta}$, and find

$$
w(y, \varepsilon, \lambda) \leq g(y, \varepsilon, \lambda)^{\frac{N+1}{\delta} y} .
$$

The corollary follows from the facts that $\frac{N+1}{\delta} y \geq(N+1)$ and, for $\varepsilon$ small enough, $g(y, \varepsilon, \lambda)<1$.

For the proof of (P2), define

$$
F(y, \varepsilon, \lambda)=w(y, \varepsilon, \lambda)^{1 / M} g(y, \varepsilon, \lambda)^{K} \int_{y_{0}}^{y} \frac{d s}{g(s, \varepsilon, \lambda)^{K}} .
$$

Lemma A.4. For all $\nu>0$, there exists a neighbourhood $V$ of $\varepsilon=\varepsilon_{0}$ such that

$$
F(y, \varepsilon, \lambda)<\nu,
$$

for all $y \in\left[y_{0}, y_{1}\right], \lambda \in \Lambda$ and $\varepsilon \in V$ (only for those $\varepsilon$ where $w$ is defined). This proves property $(\mathrm{P} 2)$ in case (a).

Proof. It is sufficient that we prove

$$
F\left(y_{0}, \varepsilon, \lambda\right)<\nu \quad \text { and } \quad\left(F(y, \varepsilon, \lambda) \geq \nu \Longrightarrow \frac{\partial F}{\partial y}(y, \varepsilon, \lambda)<0\right) .
$$

The first statement is obvious, since $F\left(y_{0}, \varepsilon, \lambda\right)=0$. To prove the second statement, assume $F(y, \varepsilon, \lambda) \geq \nu$. Then (write $g^{\prime}$ for $\frac{\partial g}{\partial y}$ ):

$$
\begin{aligned}
\frac{\partial F}{\partial y} & =w^{1 / M}\left(\frac{-1}{M g} g^{K} \int \frac{1}{g^{K}}+K g^{K-1} g^{\prime} \int \frac{1}{g^{K}}+g^{K} \frac{1}{g^{K}}\right) \\
& =w^{1 / M}+\left(\frac{-1}{M g}+\frac{K g^{\prime}}{g}\right)\left(w^{1 / M} g^{K} \int 1 g^{K}\right) \\
& =w^{1 / M}-\frac{F}{M g}\left(1-K M g^{\prime}\right) .
\end{aligned}
$$

Taking $\varepsilon$ small enough, we may assume that $g^{\prime} \leq \frac{1}{2 K M}$. Since we also know that $w$ is bounded by 1 , we have

$$
\frac{\partial F}{\partial y} \leq 1-\frac{F}{M g}\left(1-\frac{1}{2}\right) \leq 1-\frac{\nu}{2 M g} .
$$

As $g$ gets smaller, $\frac{\partial F}{\partial y}$ will become negative.

These lemmas prove properties (P1) and (P2) in the case (a) of Proposition A.1. and it was already pointed out that this is enough in view of proving Proposition A.1. Let us now adapt the proof to the case (b). A key element in the proofs of the lemmas was the fact that $g$ and $g^{\prime}$ becomes zero. In the case (b), we do not have this property uniformly in $\left[y_{0}, y_{1}\right]$, but only in the end point $y_{1}$. We will need to be more careful:

Lemma A.5. If we define $F(y, \varepsilon, \lambda):=\int_{y_{0}}^{y} \frac{d s}{g(s, \varepsilon, \lambda)}+K\left(y-y_{0}\right) \log g(y, \varepsilon, \lambda)$, then there exists a $\delta \in] y_{0}, y_{1}\left[\right.$ and a neighbourhood $V$ of $\varepsilon=\varepsilon_{0}$ such that for all $y \in\left[\delta, y_{1}\right]$,

$$
F(y, \varepsilon, \lambda) \geq F(\delta, \varepsilon, \lambda),
$$

for all $\lambda \in \Lambda$ and $\varepsilon \in V$ (only for those $\varepsilon$ where $w$ is defined.)

Proof. Completely similar to the proof of Lemma A.2. 
Corollary A.6. For all $N \in \mathbb{N}$ there exists a neighbourhood $V$ of $\varepsilon=\varepsilon_{0}$, a $C>0$ and $a \delta \in] y_{0}, y_{1}[$ such that

$$
w(y, \varepsilon, \lambda) \leq C g(y, \varepsilon, \lambda)^{N+1},
$$

for all $y \in\left[\delta, y_{1}[\right.$, for all $\lambda \in \Lambda$ and $\varepsilon \in V$ (only for those $\varepsilon$ where $w$ is defined). This proves property $(\mathrm{P} 1)$ in case $(\mathrm{b})$.

Proof. Apply the lemma to $K=\frac{N+1}{\tilde{\delta}}$, where $\tilde{\delta}$ is an arbitrary small number. There exists a $\delta \in\left[y_{0}, y_{1}\left[\right.\right.$ such that for all $y \in\left[\delta, y_{1}[\right.$,

$$
w(y, \varepsilon, \lambda) \leq g(y, \varepsilon, \lambda)^{K\left(y-y_{0}\right)} g(\delta, \varepsilon, \lambda)^{-K\left(\delta-y_{0}\right)} w(\delta, \varepsilon, \lambda) .
$$

So for $y \geq \max \left\{\delta, y_{0}+\tilde{\delta}\right\}$,

$$
w(y, \varepsilon, \lambda) \leq g(y, \varepsilon, \lambda)^{N+1} g(\delta, \varepsilon, \lambda)^{-K\left(\delta-y_{0}\right)} w(\delta, \varepsilon, \lambda) .
$$

Since $g(\delta, 0, \lambda) \neq 0$, we find that it can be bounded away from zero, which proves the corollary.

The proof of (P2) in case (b) is completely similar as in case (a), by replacing $y_{0}$ by a $\delta$ close enough to $y_{1}$.

A slight generalization is needed. The results of Proposition A.1 remain true if not $g(y, \varepsilon, \lambda)$, but $\varepsilon^{N} g(y, \varepsilon, \lambda)$ is a $C^{k}$-function for some $N>0$ :

Proposition A.7. Let $g$ be a positive (not necessarily strictly positive) $C^{0}$-function on $V \times W \times \Lambda$, where $V$ is a compact interval in $\mathbb{R}, W=\left[0, \varepsilon_{0}[\right.$ is a set of singular parameters, and $\Lambda$ is a set of regular parameters (part of a finitely dimensional vector space). Assume that $\varepsilon^{N} g$ is a $C^{k}$-function for some $N \geq 0$. Define for a fixed $y_{0} \in V$, and for all $y>y_{0}$,

$$
w(y, \varepsilon, \lambda):=\exp \left(-\int_{y_{0}}^{y} \frac{d s}{g(s, \varepsilon, \lambda)}\right), \quad \varepsilon \in W, \lambda \in \Lambda .
$$

Assume for $y_{1}>y_{0}$ that $\left(y_{1}, 0, \lambda\right)$ is in the closure of the domain of $w$. If $g(s, 0, \lambda)=$ $0, \forall s \in\left[y_{0}, y_{1}\right]$, then the function $w$ can be extended in a $C^{k}$ way to $\left(y_{1}, 0, \lambda\right)$, and in this point $w$ and all its derivatives (up to order $k$ ) are zero.

Proof. The setting is similar to the setting in Proposition A.1, and the proof can be copied after a slight change: next to the properties (P1) and (P2), we have to prove additionally

$$
w(y, \varepsilon, \lambda)=O\left(\varepsilon^{\tilde{N}}\right), \quad \forall \tilde{N}>0 .
$$

Let us explain why: to show that the properties (P1) and (P2) are sufficient to prove the smoothness in Proposition A.1, we replaced all differentials of $g$ by constantsthis is possible since all differentials are $C^{0}$. Here, we have to replace the differentials by $C / \varepsilon^{\tilde{N}}$, hence (P3) is needed. The proof of (P3) is trivial since $g \leq K \varepsilon$ for some constant $K>0$, and thus $w(y, \varepsilon, \lambda) \leq \exp \left(-\frac{y-y_{0}}{K \varepsilon}\right)$. 


\section{REFERENCES}

[BE] E. Benoît, Applications of nonstandard analysis in ordinary differential equations, NATO Adv. Sci. Inst. Ser. C Math. Phys. Sci, vol. 493, 1997, 153-182. MR1603233(99i:34075)

[BCDD] E. Benoît, J-L. Callot, F. Diener, M. Diener, Chasse au canard, I-IV, Collect. Math., vol. 32, no. 1-2 (1981). MR0643399 (85g:58062a) MR0653888 (85g:58062b) MR0653889 (85g:58062c) MR0653890(85g:58062d)

[BFSW] E. Benoît, A. Fruchard, R. Schäfke, G. Wallet, solutions surstables des équations différentielles complexes lentes-rapides à point tournant, Ann. Fac. Sci. Toulouse Math. 6, vol. 7, no. 4, 1998, 627-658. MR.1693589 (2000g:34084)

[Bon] P. Bonckaert, Partially hyperbolic fixed points with constraints, Trans. Amer. Math. Soc., vol. 348, no. 3, 1996, 997-1011. MR.1321568 (96h:58157)

[CD] M. Canalis-Durand, Caractère Gevrey du developpement formel des solutions canard de l'équation de Van der Pol, C. R. Acad. Sci. Paris Sér. A Math. 311, 1990, 27-30. MR:1062923 (91j:34084)

[CRSS] M. Canalis-Durand, J.P. Ramis, R. Schäfke, Y. Sibuya, Gevrey solutions of singularly perturbed differential equations, J. Reine Angew. Math., no. 588, 2000, 95-129. MR:1739408 (2000m:34207)

[DE] A. Delcroix, A tool for the local study of slow-fast vector fields: the zoom, Lecture Notes in Mathematics, no. 1493 (1991). MR 1167002 (93g:34083)

[D] F. Dumortier, Techniques in the theory of local bifurcations: blowup, normal forms, nilpotent bifurcations, singular perturbations (notes written with B. Smits), in "Bifurcations and periodic orbits and vector fields" (ed. D. Schmoliuk), NATO ASI series, series C: Math. and Phys. Sciences, vol. 408, 1993, 19-73. MR1258518 (94j:58123)

[DR] F. Dumortier and R. Roussarie, Canard cycles and Center manifolds, Memoirs of the Amer. Math. Soc., vol. 121, no. 577, 1996. MR1327208 (96k:34113)

[DR2] F. Dumortier and R. Roussarie, Multiple canard cycles in generalized Liénard equations, Journal of differential equations, vol. 174, no. 1, 2001, 1-29. MR1844521 (2002k:34076)

[DR3] F. Dumortier and R. Roussarie, Geometric singular perturbation theory beyond normal hyperbolicity, in "Multiple-time-scale dynamical systems", ed. C.K.R.T. Jones and A. Khibnik, IMA Volumes in Mathematics and its Applications, vol. 122, SpringerVerlag, 2000, 29-64. MR1846572 (2002f:37035)

[ECK] W. Eckhaus, Asymptotic Analysis of singular perturbations, Studies in Mathematics and its applications, vol. 9, 1979. MR0553107 (81a:34048)

[ECK2] W. Eckhaus, Fundamental concepts of matching, SIAM rev., vol. 36, no. 3, 1994, 431439. MR1292646 (95g:34076)

[FE] N. Fenichel, Geometric Singular Perturbation Theory for ordinary differential equations, J. Differential Equations, vol. 31, no. 1, 1979, 53-98. MR0524817(80m:58032)

[HPS] M. Hirsch, C. Pugh, M. Shub, Invariant Manifolds, Lecture Notes in Mathematics, 583, Springer-Verlag, 1977. MR0501173 (58:18595)

[JKK] C.K.R.T. Jones, T.J. Kaper, N. Kopell, Tracking invariant manifolds up to exponentially small errors, SIAM. J. Math. Anal, vol. 27, no. 2, 1996, 558-577. MR.1377489|(97a:58167)

[K] A. Kelley, The stable, center-stable, center, center-unstable and unstable manifolds, Appendix C in R. Abraham, J. Robbin: Transversal mappings and flows, Benjamin, New York, 1967. MR0221044 (36:4096)

[KS1] M. Krupa, P. Szmolyan, Relaxation oscillation and canard explosion, J. Differential Equations, vol. 174, no. 2, 2001, 312-368. MR.1846739 (2002g:34122)

[KS2] M. Krupa, P. Szmolyan, Geometric analysis of the singularly perturbed planar fold, in "Multiple-time-scale dynamical systems", IMA Volumes in Mathematics and its Applications, vol. 122, 2001, 89-116. MR 1846574 (2002j:34089)

[KS3] M. Krupa, P. Szmolyan, Extending geometric singular perturbation theory to nonhyperbolic points-fold and canard points in two dimensions, SIAM J. Math. Anal., vol. 33, no. 2, 2001, 286-314. MR 1857972 (2002g:34117)

[KS4] M. Krupa, P. Szmolyan, Extending slow manifolds near transcritical and pitchfork singularities, Nonlinearity, vol. 14, no. 6, 2001, 1473-1491. MR.1867088 (2002k:34112)

[MKKR] E.F. Mishchenko, Yu.S. Kolesov, A.Yu. Kolesov, N.Kh. Rozov, Asymptotic Methods in Singularly Perturbed Systems, Monographs in Contemporary Mathematics, Consultants Bureau New York, 1994. MR 1320052 (96h:34109) 
[PA] D. Panazzolo, On the existence of canard solutions, Publ. Mat., vol. 44, no. 2, 2000, 503-592. MR.1800821 (2002f:34125)

[PO] L.S. Pontryagin, Asymptotic behavior of the solutions of systems of differential equations with a small parameter in the higher derivatives, Amer. Math. Soc. Transl. (2), vol. 18, 1961, 295-319. MR124591 (23:A1903)

[VDP] B. Van Der Pol, On relaxation oscillations, Phil. Mag., vol. 2, 1926, 978-992.

[WA] W. Wasow, Asymptotic expansions for ordinary differential equations, reprint, Dover Publications Inc., New York, 1987. MR0919406 (88i:34003)

Departement Wiskunde, Natuurkunde, Informatica, Dynamical Systems, Hasselt University, Agoralaan, Gebouw D, B-3590 Diepenbeek, Belgium

E-mail address: peter.demaesschalck@uhasselt.be

Departement Wiskunde, Natuurkunde, Informatica, Dynamical Systems, Hasselt University, Agoralaan, Gebouw D, B-3590 Diepenbeek, Belgium

E-mail address: freddy.dumortier@uhasselt.be 